CERN-TH/96-19

SHEP 96-03

hep-ph/9603202

\title{
Spectator Effects in Inclusive Decays of Beauty Hadrons
}

\author{
M. Neubert and C.T. Sachrajda* \\ Theory Division, CERN, CH-1211 Geneva 23, Switzerland
}

\begin{abstract}
We present a model-independent study of spectator effects, which are responsible for the lifetime differences between beauty hadrons. These effects can be parametrized in terms of hadronic matrix elements of four four-quark operators. For $B$ mesons, the coefficients of the non-factorizable operators turn out to be much larger than those of the factorizable ones, limiting considerably the usefulness of the vacuum insertion approximation. Non-factorizable contributions to the lifetime ratio $\tau\left(B^{-}\right) / \tau\left(B_{d}\right)$ could naturally be of order 10-20\%, and not even the sign of these contributions can be predicted at present. In the case of the $\Lambda_{b}$ baryon, heavy-quark symmetry is used to reduce the number of independent matrix elements from four to two. In order to explain the large deviation from unity in the experimental result for $\tau\left(\Lambda_{b}\right) / \tau\left(B_{d}\right)$, it is necessary that these baryon matrix elements be much larger than those estimated in quark models. We have also reexamined the theoretical predictions for the semileptonic branching ratio of $B$ mesons and charm counting, finding that, given the present theoretical and experimental uncertainties, there is no significant discrepancy with experiment.
\end{abstract}

(Revised Version)

CERN-TH/96-19

September 1996 
*On leave from the Department of Physics, University of Southampton, Southampton SO17 1BJ, UK 


\section{Introduction}

In this paper we study "spectator effects" in inclusive decays of beauty hadrons. These effects involve the participation of the light constituents in the decay and thus contribute to the differences in the decay widths and lifetimes of different species of beauty hadrons. Indeed, one of our goals is to understand theoretically the experimental results for the lifetime ratios [四]:

$$
\begin{aligned}
& \frac{\tau\left(B^{-}\right)}{\tau\left(B_{d}\right)}=1.02 \pm 0.04 \\
& \frac{\tau\left(B_{s}\right)}{\tau\left(B_{d}\right)}=1.01 \pm 0.07 \\
& \frac{\tau\left(\Lambda_{b}\right)}{\tau\left(B_{d}\right)}=0.78 \pm 0.05 .
\end{aligned}
$$

Here $\tau\left(B_{s}\right)$ refers to the average $B_{s}$-meson lifetime. Our study is performed in the framework of the heavy-quark expansion, in which these ratios are computed as series in inverse powers of the mass of the $b$ quark [2]- [5] (for recent reviews, see refs. [6, 7]). The leading term of this expansion corresponds to the decay of a free $b$ quark. This term is universal, contributing equally to the lifetimes of all beauty hadrons. Remarkably, the first correction to this result is of order $\left(\Lambda_{\mathrm{QCD}} / m_{b}\right)^{2}$ 3, 4. This leads to the theoretical predictions (see section 2 below)

$$
\begin{aligned}
& \frac{\tau\left(B^{-}\right)}{\tau\left(B_{d}\right)}=1+O\left(1 / m_{b}^{3}\right) \\
& \frac{\tau\left(B_{s}\right)}{\tau\left(B_{d}\right)}=(1.00 \pm 0.01)+O\left(1 / m_{b}^{3}\right) \\
& \frac{\tau\left(\Lambda_{b}\right)}{\tau\left(B_{d}\right)}=0.98+O\left(1 / m_{b}^{3}\right) .
\end{aligned}
$$

The deviation from this expectation for $\tau\left(\Lambda_{b}\right) / \tau\left(B_{d}\right)$ is striking, and is the principle motivation for this study.

Spectator effects, i.e. contributions from decays in which a light constituent quark also participates in the weak process, have first been considered in refs. [8][10]. For decays of heavy particles, these effects are strongly suppressed due to the need for the $b$ quark and a light quark in the heavy hadron to be close together (i.e. from a factor of the "wave-function at the origin"). As the portion of the volume that the $b$ quark occupies inside the hadron is of order $\left(\Lambda_{\mathrm{QCD}} / m_{b}\right)^{3}$, spectator effects appear only at third order in the heavy-quark expansion, and it might seem safe to neglect them altogether. However, as a result of the difference in the phase-space for $2 \rightarrow 2$-body reactions as compared to $1 \rightarrow 3$-body decays, these effects are enhanced by a factor of order $16 \pi^{2}$. It is conceivable that they could be larger than the terms of order $\left(\Lambda_{\mathrm{QCD}} / m_{b}\right)^{2}$ included in (2). Moreover, spectator 
effects explicitly differentiate between different species of beauty hadrons. In order to understand the structure of lifetime differences, it is therefore important to reconsider the analysis of such effects. The striking experimental result for the short $\Lambda_{b}$ lifetime gives an additional motivation to such a study.

Previous studies of spectator effects in the decays of beauty hadrons [6] were performed using the formalism developed in refs. [8]-10] and made two simplifying assumptions: first, the hadronic matrix elements of these operators were estimated employing the vacuum insertion approximation [11] for mesons and quark models [8, 12] for baryons, and secondly the mass of the charm quark was neglected in the calculation of the coefficients of the four-quark operators in the heavy-quark expansion. In order to explore the acceptable range of theoretical predictions, we do not impose factorization or quark-model approximations on the hadronic matrix elements. Instead, we parametrize them by a set of hadronic parameters and see how the lifetime ratios depend upon them. One of our main conclusions is that only a detailed field-theoretic calculation of the relevant matrix elements can lead to reliable predictions. In addition, we derive the exact expressions for the coefficients as functions of the charm-quark mass.

The semileptonic branching ratio of $B$ mesons has also received considerable attention. For many years it appeared that the theoretical predictions for this quantity [13]-15] lay above the measured value. More recently, the theoretical predictions have been refined by including exact expressions for the $O\left(\alpha_{s}\right)$ corrections [16]. Using the results of these calculations, we present a new analysis of the semileptonic branching ratio $B_{\mathrm{SL}}$ and the average charm multiplicity $n_{c}$ in $B$ decays. We find that the freedom in the choice of the renormalization scale (which reflects the ignorance of higher-order perturbative corrections) allows us to obtain consistent predictions for both quantities simultaneously. We also calculate the spectator contributions to $B_{\mathrm{SL}}$ and $n_{c}$ and show that they could change the semileptonic branching ratio by an amount of order $1 \%$, whereas their effect on $n_{c}$ is negligible.

In section 2, we discuss the heavy-quark expansion for inclusive decay rates, and we present our results for the contributions arising from spectator effects. In section 3, we introduce a set of hadronic parameters defined in terms of the relevant four-quark operator matrix elements between $B$-meson and $\Lambda_{b}$-baryon states. Heavy-quark symmetry is used to derive some new relations between the baryonic matrix elements of these operators. In section 1 , we then discuss the phenomenological implications of our results for the understanding of beauty lifetimes. We also present a critical discussion of previous estimates of spectator effects based on the factorization approximation. A detailed discussion of the semileptonic branching ratio of $B$ mesons is presented in section 5 . Section 6 contains the conclusions. The renormalization of the operators and parameters describing the spectator effects, and the behaviour of these parameters with respect to the large- $N_{c}$ limit, are discussed in appendix $\mathrm{A}$, while appendix $\mathrm{B}$ contains details about the calculation of the semileptonic branching ratio. 
The reader who is primarily interested in the phenomenological implications of our analysis can omit sections 2 and 3 and proceed directly to sections 1 , 5 and 6, which are written in a self-contained way.

\section{Heavy-quark expansion}

Inclusive decay rates, which determine the probability of the decay of a particle into the sum of all possible final states with a given set of quantum numbers $\{f\}$, have two advantages from the theoretical point of view: first, bound-state effects related to the initial state can be accounted for in a systematic way using the heavy-quark expansion; secondly, the fact that the final state consists of a sum over many hadronic channels eliminates bound-state effects related to the properties of individual hadrons. This second feature is based on the hypothesis of quark-hadron duality, i.e. the assumption that cross sections and decay rates are calculable in QCD after a "smearing" procedure has been applied [17]. We shall not discuss this hypothesis here; however, if after the non-perturbative evaluation of the spectator effects discussed in our analysis there remain significant discrepancies between theory and experiment (for the lifetime ratio $\tau\left(\Lambda_{b}\right) / \tau\left(B_{d}\right)$, in particular), one may have to seriously question the assumption of duality. A recent study of inclusive $B$ decays, in which duality violations are invoked to add non-perturbative contributions of order $\Lambda_{\mathrm{QCD}} / m_{b}$ not present in the heavy-quark expansion, can be found in ref. [18].

Using the optical theorem, the inclusive decay width of a hadron $H_{b}$ containing a $b$ quark can be written as the forward matrix element of the imaginary part of the transition operator $\mathbf{T}$,

$$
\Gamma\left(H_{b} \rightarrow X\right)=\frac{1}{m_{H_{b}}} \operatorname{Im}\left\langle H_{b}|\mathbf{T}| H_{b}\right\rangle=\frac{1}{2 m_{H_{b}}}\left\langle H_{b}|\boldsymbol{\Gamma}| H_{b}\right\rangle,
$$

where $\mathbf{T}$ is given by

$$
\mathbf{T}=i \int \mathrm{d}^{4} x T\left\{\mathcal{L}_{\text {eff }}(x), \mathcal{L}_{\text {eff }}(0)\right\} .
$$

For the case of semileptonic and non-leptonic decays, the effective weak Lagrangian, renormalized at the scale $\mu=m_{b}$, is

$$
\begin{aligned}
\mathcal{L}_{\text {eff }}=-\frac{4 G_{F}}{\sqrt{2}} V_{c b} & \left\{c_{1}\left(m_{b}\right)\left[\bar{d}_{L}^{\prime} \gamma_{\mu} u_{L} \bar{c}_{L} \gamma^{\mu} b_{L}+\bar{s}_{L}^{\prime} \gamma_{\mu} c_{L} \bar{c}_{L} \gamma^{\mu} b_{L}\right]\right. \\
& +c_{2}\left(m_{b}\right)\left[\bar{c}_{L} \gamma_{\mu} u_{L} \bar{d}_{L}^{\prime} \gamma^{\mu} b_{L}+\bar{c}_{L} \gamma_{\mu} c_{L} \bar{s}_{L}^{\prime} \gamma^{\mu} b_{L}\right] \\
& \left.+\sum_{\ell=e, \mu, \tau} \bar{\ell}_{L} \gamma_{\mu} \nu_{\ell} \bar{c}_{L} \gamma^{\mu} b_{L}\right\}+ \text { h.c. }
\end{aligned}
$$


where $q_{L}=\frac{1}{2}\left(1-\gamma_{5}\right) q$ denotes a left-handed quark field, $d^{\prime}=d \cos \theta_{c}+s \sin \theta_{c}$ and $s^{\prime}=s \cos \theta_{c}-d \sin \theta_{c}$ are the Cabibbo-rotated down- and strange-quark fields $\left(\sin \theta_{c} \simeq 0.2205\right)$, and we have neglected $b \rightarrow u$ transitions. The Wilson coefficients $c_{1}$ and $c_{2}$ take into account the QCD corrections arising from the fact that the effective Lagrangian is written at a renormalization scale $\mu=m_{b}$ rather than $m_{W}$. They can be calculated in perturbation theory. The combinations $c_{ \pm}=c_{1} \pm c_{2}$ have a multiplicative evolution under change of the renormalization scale. To leading order, they are given by [19]-21]

$$
c_{ \pm}\left(m_{b}\right)=\left(\frac{\alpha_{s}\left(m_{W}\right)}{\alpha_{s}\left(m_{b}\right)}\right)^{a_{ \pm}}, \quad a_{-}=-2 a_{+}=-\frac{12}{33-2 n_{f}} .
$$

In the numerical analysis we shall take the values $c_{+}\left(m_{b}\right) \simeq 0.86$ and $c_{-}\left(m_{b}\right)=$ $1 / c_{+}^{2}\left(m_{b}\right) \simeq 1.35$, corresponding to $\alpha_{s}\left(m_{Z}\right)=0.117$.

Since the energy release in the decay of a $b$ quark is large, it is possible to construct an Operator Product Expansion (OPE) for the bilocal transition operator (田), in which it is expanded as a series of local operators with increasing dimension, whose coefficients contain inverse powers of the $b$-quark mass. The operator with the lowest dimension is $\bar{b} b$. There is no independent operator with dimension four, since the only candidate, $\bar{b} i \not D b$, can be reduced to $\bar{b} b$ by using the equations of motion [3, [4. The first new operator is $\bar{b} g_{s} \sigma_{\mu \nu} G^{\mu \nu} b$ and has dimension five. Thus, any inclusive decay rate of a hadron $H_{b}$ can be written in the form

$$
\Gamma\left(H_{b} \rightarrow X_{f}\right)=\frac{G_{F}^{2} m_{b}^{5}}{192 \pi^{3}} \frac{1}{2 m_{H_{b}}}\left\{c_{3}^{f}\left\langle H_{b}|\bar{b} b| H_{b}\right\rangle+c_{5}^{f} \frac{\left\langle H_{b}\left|\bar{b} g_{s} \sigma_{\mu \nu} G^{\mu \nu} b\right| H_{b}\right\rangle}{m_{b}^{2}}+\ldots\right\},
$$

where $c_{n}^{f}$ are calculable coefficient functions (which also contain the relevant CKM matrix elements) depending on the quantum numbers $\{f\}$ of the final state. For semileptonic and non-leptonic decays, the coefficients $c_{3}^{f}$ have been calculated at one-loop order [22, 23, 16], and the coefficients $c_{5}^{f}$ at tree level [3, 24].

In the next step, the forward matrix elements of the local operators in the OPE are systematically expanded in inverse powers of the $b$-quark mass, using the heavy-quark effective theory (HQET) [25]. One finds [3, [4]

$$
\begin{aligned}
\frac{1}{2 m_{H_{b}}}\left\langle H_{b}|\bar{b} b| H_{b}\right\rangle & =1-\frac{\mu_{\pi}^{2}\left(H_{b}\right)-\mu_{G}^{2}\left(H_{b}\right)}{2 m_{b}^{2}}+O\left(1 / m_{b}^{3}\right), \\
\frac{1}{2 m_{H_{b}}}\left\langle H_{b}\left|\bar{b} g_{s} \sigma_{\mu \nu} G^{\mu \nu} b\right| H_{b}\right\rangle & =2 \mu_{G}^{2}\left(H_{b}\right)+O\left(1 / m_{b}\right),
\end{aligned}
$$

where $\mu_{\pi}^{2}\left(H_{b}\right)$ and $\mu_{G}^{2}\left(H_{b}\right)$ parametrize the matrix elements of the kinetic-energy and the chromo-magnetic operators, respectively. The purpose of doing this expansion is that whereas the matrix elements in (7) contain an implicit dependence on the $b$-quark mass, the parameters appearing on the right-hand side of (8) are 
independent of $m_{b}$ (modulo logarithms). These parameters can be determined, to some extent, from the spectrum of heavy hadron states. Below we shall need the values

$$
\begin{aligned}
\mu_{\pi}^{2}\left(\Lambda_{b}\right)-\mu_{\pi}^{2}(B) & =-(0.01 \pm 0.03) \mathrm{GeV}^{2}, \\
\mu_{G}^{2}(B) & =\frac{3}{4}\left(m_{B^{*}}^{2}-m_{B}^{2}\right) \simeq 0.36 \mathrm{GeV}^{2}, \\
\mu_{G}^{2}\left(\Lambda_{b}\right) & =0 .
\end{aligned}
$$

The difference $\mu_{\pi}^{2}\left(\Lambda_{b}\right)-\mu_{\pi}^{2}(B)$ can be extracted from the mass formula [6, 7]

$$
\left(m_{\Lambda_{b}}-m_{\Lambda_{c}}\right)-\left(\bar{m}_{B}-\bar{m}_{D}\right)=\left[\mu_{\pi}^{2}(B)-\mu_{\pi}^{2}\left(\Lambda_{b}\right)\right]\left(\frac{1}{2 m_{c}}-\frac{1}{2 m_{b}}\right)+O\left(1 / m_{Q}^{2}\right),
$$

where $\bar{m}_{B}=\frac{1}{4}\left(m_{B}+3 m_{B^{*}}\right)$ and $\bar{m}_{D}=\frac{1}{4}\left(m_{D}+3 m_{D^{*}}\right)$ denote the spin-averaged meson masses. With $m_{\Lambda_{b}}=(5625 \pm 6) \mathrm{MeV}$ [26], this relation leads to the value quoted above.

To order $1 / m_{b}^{2}$ in the heavy-quark expansion, the lifetime ratio for two beauty hadrons is given by

$$
\frac{\tau\left(H_{b}^{(1)}\right)}{\tau\left(H_{b}^{(2)}\right)}=1+\frac{\mu_{\pi}^{2}\left(H_{b}^{(1)}\right)-\mu_{\pi}^{2}\left(H_{b}^{(2)}\right)}{2 m_{b}^{2}}+c_{G} \frac{\mu_{G}^{2}\left(H_{b}^{(1)}\right)-\mu_{G}^{2}\left(H_{b}^{(2)}\right)}{m_{b}^{2}}+O\left(1 / m_{b}^{3}\right),
$$

where $c_{G} \simeq 1.2$ can be obtained using the results of refs. [3, 24]. Using then the values given in (9), and assuming that in the case of the $B_{s}$ meson $\mathrm{SU}(3)$ breaking effects in the values of the matrix elements are of order $20 \%$, we arrive at the predictions given in (2). Note that in taking a ratio of lifetimes, theoretical uncertainties related to the values of the $b$-quark mass (including renormalon ambiguities) and CKM elements cancel to a large extent. It is for this reason that we restrict our discussion to the calculation of ratios of lifetimes and decay rates.

The first two terms in the heavy-quark expansion (7) arise from decays in which the (light) spectator quarks interact only softly. Additional contributions of this type appear at the next order through gluonic operators of dimension six, such as $\bar{b} \gamma_{\mu}\left(i D_{\nu} G^{\mu \nu}\right) b$. Since matrix elements of these operators are blind to the flavour of the spectator quarks, they can be safely neglected in our analysis. "Hard" spectator effects manifest themselves first in the matrix elements of four-quark operators of dimension six. Some examples of the corresponding contributions to the transition operator $\mathbf{T}$ are shown in figure 11. They only appear in the heavy-quark expansion of non-leptonic decay rates.7 Since these contributions arise from one-loop rather than two-loop diagrams, they receive a phase-space enhancement factor of order $16 \pi^{2}$ relative to the other terms in the heavy-quark expansion.

\footnotetext{
${ }^{1}$ This is no longer true if $b \rightarrow u$ transitions or the decays of the $B_{c}$ meson are considered.
} 


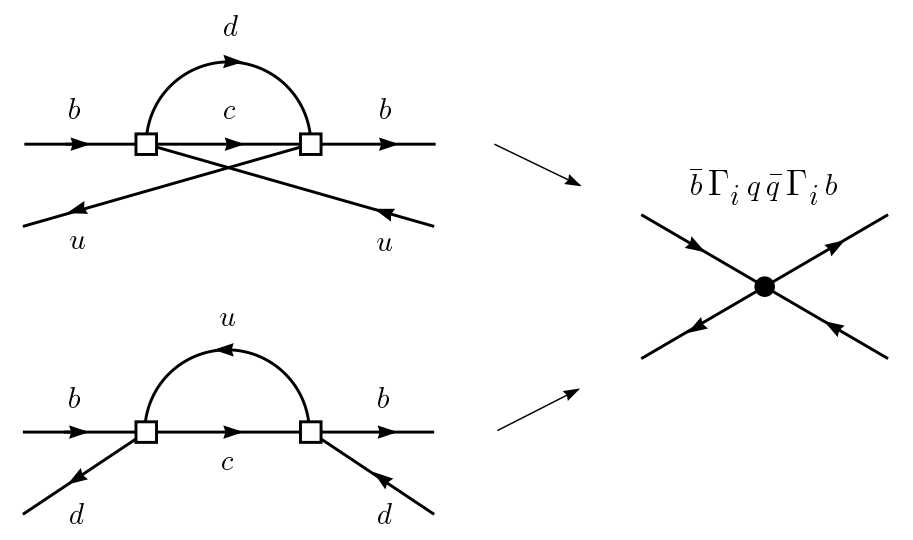

Figure 1: Spectator contributions to the transition operator $\mathbf{T}$ (left), and the corresponding operator in the OPE (right). Here $\Gamma_{i}$ denotes some combination of Dirac and colour matrices.

We have calculated the coefficients of the corresponding four-quark operators at tree level, including for the first time the dependence on the mass of the charm quark. This extends the results obtained in ref. [6]. We find that the corresponding contributions to the non-leptonic widths of mesons and baryons containing a $b$ quark are given by the matrix elements of the local operator

$$
\begin{aligned}
\boldsymbol{\Gamma}_{\mathrm{spec}}=\frac{2 G_{F}^{2} m_{b}^{2}}{\pi}\left|V_{c b}\right|^{2}(1-z)^{2}\left\{\left(2 c_{1} c_{2}+\frac{1}{N_{c}}\left(c_{1}^{2}+c_{2}^{2}\right)\right) O_{V-A}^{u}+2\left(c_{1}^{2}+c_{2}^{2}\right) T_{V-A}^{u}\right\} \\
-\frac{2 G_{F}^{2} m_{b}^{2}}{3 \pi}\left|V_{c b}\right|^{2}(1-z)^{2}\left\{\left(2 c_{1} c_{2}+\frac{1}{N_{c}} c_{1}^{2}+N_{c} c_{2}^{2}\right)\left[\left(1+\frac{z}{2}\right) O_{V-A}^{d^{\prime}}-(1+2 z) O_{S-P}^{d^{\prime}}\right]\right. \\
\left.+2 c_{1}^{2}\left[\left(1+\frac{z}{2}\right) T_{V-A}^{d^{\prime}}-(1+2 z) T_{S-P}^{d^{\prime}}\right]\right\} \\
-\frac{2 G_{F}^{2} m_{b}^{2}}{3 \pi}\left|V_{c b}\right|^{2} \sqrt{1-4 z}\left\{\left(2 c_{1} c_{2}+\frac{1}{N_{c}} c_{1}^{2}+N_{c} c_{2}^{2}\right)\left[(1-z) O_{V-A}^{s^{\prime}}-(1+2 z) O_{S-P}^{s^{\prime}}\right]\right. \\
\left.+2 c_{1}^{2}\left[(1-z) T_{V-A}^{s^{\prime}}-(1+2 z) T_{S-P}^{s^{\prime}}\right]\right\},
\end{aligned}
$$

where $z=m_{c}^{2} / m_{b}^{2}$, and $N_{c}=3$ is the number of colours. The local four-quark operators appearing in this expression are defined by

$$
\begin{aligned}
O_{V-A}^{q} & =\bar{b}_{L} \gamma_{\mu} q_{L} \bar{q}_{L} \gamma^{\mu} b_{L}, \\
O_{S-P}^{q} & =\bar{b}_{R} q_{L} \bar{q}_{L} b_{R}, \\
T_{V-A}^{q} & =\bar{b}_{L} \gamma_{\mu} t_{a} q_{L} \bar{q}_{L} \gamma^{\mu} t_{a} b_{L}, \\
T_{S-P}^{q} & =\bar{b}_{R} t_{a} q_{L} \bar{q}_{L} t_{a} b_{R},
\end{aligned}
$$

where $t_{a}=\lambda_{a} / 2$ are the generators of colour $\mathrm{SU}(3)$. For dimensional reasons, $\boldsymbol{\Gamma}_{\text {spec }}$ is proportional to $m_{b}^{2}$ rather than $m_{b}^{5}$, in accordance with the fact that 
spectator effects contribute at third order in the heavy-quark expansion. The first term in (12) arises from the upper diagram in figure 1, whereas the second and third terms come from the contributions of the lower diagram with a $c \bar{u}$ and $c \bar{c}$ quark pair in the loop. We note that in the limit $z=0$ our results agree with ref. [6], and with the corresponding expression derived for the lifetimes of charm hadrons in refs. [8]- 10 .

The operators in (13) are renormalized at the scale $m_{b}$, which will be implicit in our discussion below. This choice has the advantage that logarithms of the type $\left[\alpha_{s} \ln \left(m_{b} / \mu_{\text {had }}\right)\right]^{n}$, where $\mu_{\text {had }}$ is a typical hadronic scale, reside entirely in the hadronic matrix elements of the renormalized operators. Using the renormalization-group equations, the expressions presented in this paper can be rewritten in terms of operators renormalized at any other scale. However, at present the scale dependence of the renormalized operators below the scale $m_{b}$ is known only to leading logarithmic order [27, 28]. It is discussed in detail in appendix A. Since here we shall treat the matrix elements as unknown parameters, we can avoid all uncertainties related to the operator evolution by working at the scale $m_{b}$.

The hadronic matrix elements of the four-quark operators in (13) contain the non-perturbative physics of the spectator contributions to inclusive decays of beauty hadrons. However, the same operators also contribute to the decay of the $b$ quark, through tadpole diagrams in which the light-quark fields are contracted in a loop. These "non-spectator" contributions are independent of the flavour of the light quark $q$ and thus contribute equally to the decay widths of all beauty hadrons. They are not of interest to our discussion here. In order to isolate the true spectator effects, we shall implicitly assume a normal ordering of the fourquark operators, which has the effect of subtracting tadpole-like diagrams. In practice, this is equivalent to choosing a particular renormalization prescription for the operators. Alternatively, one may isolate the spectator effects by considering light-quark flavour non-singlet combinations of the operators; thus, for example, for matrix elements between $B_{d}$ states one could take $\left(O^{d}-O^{u}\right)$ and $\left(T^{d}-T^{u}\right)$ instead of $O^{d}$ and $T^{d}$.

\section{Parametrization of the matrix elements}

In previous analyses [6], [8]-[10], the hadronic matrix elements of the four-quark operators in (13) have been estimated making simplifying assumptions. Here we shall avoid such assumptions and, without any loss of generality, express the relevant matrix elements in terms of a set of hadronic parameters. Clearly, such an approach has less predictive power; however, it does allow us to find the range of predictions that can be made in a model-independent way. In view of the apparent discrepancy between theory and experiment for the $\Lambda_{b}$ lifetime, we find it worth while to question the model-dependent assumptions made in earlier 
analyses. Ultimately, the relevant hadronic parameters may be calculated using some field-theoretic approach such as lattice gauge theory or QCD sum rules. It has also been suggested that combinations of these parameters may be extracted from a precise measurement of the lepton spectrum in the endpoint region of semileptonic $B$ decays, or from a study of spectator effects in charm decays [29].

\subsection{Mesonic matrix elements}

For matrix elements of the four-quark operators between $B$-meson states, we define parameters $B_{i}$ and $\varepsilon_{i}$ such that:

$$
\begin{aligned}
& \frac{1}{2 m_{B_{q}}}\left\langle B_{q}\left|O_{V-A}^{q}\right| B_{q}\right\rangle \equiv \frac{f_{B_{q}}^{2} m_{B_{q}}}{8} B_{1} \\
& \frac{1}{2 m_{B_{q}}}\left\langle B_{q}\left|O_{S-P}^{q}\right| B_{q}\right\rangle \equiv \frac{f_{B_{q}}^{2} m_{B_{q}}}{8} B_{2} \\
& \frac{1}{2 m_{B_{q}}}\left\langle B_{q}\left|T_{V-A}^{q}\right| B_{q}\right\rangle \equiv \frac{f_{B_{q}}^{2} m_{B_{q}}}{8} \varepsilon_{1} \\
& \frac{1}{2 m_{B_{q}}}\left\langle B_{q}\left|T_{S-P}^{q}\right| B_{q}\right\rangle \equiv \frac{f_{B_{q}}^{2} m_{B_{q}}}{8} \varepsilon_{2}
\end{aligned}
$$

This definition is inspired by the vacuum insertion (or factorization) approximation [11], according to which the matrix elements of four-quark operators are evaluated by inserting the vacuum inside the current products. This leads to

$$
\begin{aligned}
& \left\langle B_{q}\left|O_{V-A}^{q}\right| B_{q}\right\rangle=\left(\frac{m_{b}+m_{q}}{m_{B}}\right)^{2}\left\langle B_{q}\left|O_{S-P}^{q}\right| B_{q}\right\rangle=\frac{f_{B_{q}}^{2} m_{B_{q}}^{2}}{4}, \\
& \left\langle B_{q}\left|T_{V-A}^{q}\right| B_{q}\right\rangle=\left\langle B_{q}\left|T_{S-P}^{q}\right| B_{q}\right\rangle=0,
\end{aligned}
$$

where $f_{B_{q}}$ is the decay constant of the $B_{q}$ meson, defined as

$$
\left\langle 0\left|\bar{q} \gamma^{\mu} \gamma_{5} b\right| B_{q}(p)\right\rangle=i f_{B_{q}} p^{\mu}
$$

Hence, the factorization approximation corresponds to setting $B_{i}=1$ and $\varepsilon_{i}=0$ at some scale $\mu$ (which in general will be different from our adopted choice $\mu=$ $m_{b}$ ), where the approximation is believed to be valid. The exact values of the hadronic parameters are not yet known. However, as discussed in appendix A, in the large- $N_{c}$ limit

$$
B_{i}=O(1), \quad \varepsilon_{i}=O\left(1 / N_{c}\right) .
$$

An estimate of the parameters $\varepsilon_{i}$ using QCD sum rules has been obtained by Chernyak, who finds that $\varepsilon_{1} \approx-0.15$ and $\varepsilon_{2} \approx 0$ [30]. 
In terms of the parameters $B_{i}$ and $\varepsilon_{i}$, the matrix elements of the operator $\Gamma_{\text {spec }}$ in (12) are:

$$
\begin{aligned}
& \frac{1}{2 m_{B}}\left\langle B^{-}\left|\Gamma_{\text {spec }}\right| B^{-}\right\rangle=\Gamma_{0} \eta_{\text {spec }}(1-z)^{2}\left\{\left(2 c_{+}^{2}-c_{-}^{2}\right) B_{1}+3\left(c_{+}^{2}+c_{-}^{2}\right) \varepsilon_{1}\right\}, \\
& \frac{1}{2 m_{B}}\left\langle B_{d}\left|\Gamma_{\text {spec }}\right| B_{d}\right\rangle \\
& =-\Gamma_{0} \eta_{\text {spec }}(1-z)^{2} \cos ^{2} \theta_{c}\left\{\frac{1}{3}\left(2 c_{+}-c_{-}\right)^{2}\left[\left(1+\frac{z}{2}\right) B_{1}-(1+2 z) B_{2}\right]\right. \\
& \left.\quad+\frac{1}{2}\left(c_{+}+c_{-}\right)^{2}\left[\left(1+\frac{z}{2}\right) \varepsilon_{1}-(1+2 z) \varepsilon_{2}\right]\right\} \\
& \quad-\Gamma_{0} \eta_{\text {spec }} \sqrt{1-4 z} \sin ^{2} \theta_{c}\left\{\frac{1}{3}\left(2 c_{+}-c_{-}\right)^{2}\left[(1-z) B_{1}-(1+2 z) B_{2}\right]\right. \\
& \left.\quad+\frac{1}{2}\left(c_{+}+c_{-}\right)^{2}\left[(1-z) \varepsilon_{1}-(1+2 z) \varepsilon_{2}\right]\right\}
\end{aligned}
$$

where $c_{ \pm}=c_{1} \pm c_{2}$, and

$$
\Gamma_{0}=\frac{G_{F}^{2} m_{b}^{5}}{192 \pi^{3}}\left|V_{c b}\right|^{2}, \quad \eta_{\mathrm{spec}}=16 \pi^{2} \frac{f_{B}^{2} m_{B}}{m_{b}^{3}} .
$$

The spectator contribution to the width of the $B_{s}$ meson is obtained from that of the $B_{d}$ meson by the replacements $\sin \theta_{c} \leftrightarrow \cos \theta_{c}$ and $f_{B}, m_{B} \rightarrow f_{B_{s}}, m_{B_{s}}$. Of course, the values of the parameters $B_{i}$ and $\varepsilon_{i}$ for the $B_{s}$ meson will also differ from those for the $B_{d}$ meson due to $\mathrm{SU}(3)$-breaking effects.

Two remarks are in order regarding the result (18). The first concerns the expected order of magnitude of the spectator contributions to the total decay width of a $B$ meson. At leading order in the heavy-quark expansion, the total width of a beauty hadron is $\Gamma_{\text {tot }} \simeq 3.7 \times \Gamma_{0}$, where the numerical factor arises from the phase-space contributions of the semileptonic and non-leptonic channels (we use $z=0.085$ ) [3, 24]. It follows that

$$
\frac{\Gamma_{\mathrm{spec}}}{\Gamma_{\mathrm{tot}}} \sim \frac{\eta_{\mathrm{spec}}}{4} \sim\left(\frac{2 \pi f_{B}}{m_{B}}\right)^{2} \sim 5 \% .
$$

The second remark concerns the structure of the coefficients in (18). Given that $c_{+} \simeq 0.86$ and $c_{-} \simeq 1.35$, one observes that the coefficients of the colour singlet-singlet operators are one to two orders of magnitude smaller than those of the colour octet-octet operators. This implies that at the scale $m_{b}$ even small deviations from the factorization approximation can have a sizeable impact on the results. 


\subsection{Baryonic matrix elements}

Next we study the matrix elements of the four-quark operators between $\Lambda_{b}$-baryon states. Since the $\Lambda_{b}$ is an iso-singlet, the matrix elements of the operators with $q=u$ or $d$ are the same, and below we drop this label. In the case of baryons, we find it convenient to use the colour identity

$$
\left(t_{a}\right)_{\alpha \beta}\left(t_{a}\right)_{\gamma \delta}=\frac{1}{2} \delta_{\alpha \delta} \delta_{\gamma \beta}-\frac{1}{2 N_{c}} \delta_{\alpha \beta} \delta_{\gamma \delta}
$$

to rewrite $T=-\frac{1}{6} O+\frac{1}{2} \widetilde{O}$ and introduce the operators $(i, j$ are colour indices)

$$
\widetilde{O}_{V-A}=\bar{b}_{L}^{i} \gamma_{\mu} q_{L}^{j} \bar{q}_{L}^{j} \gamma^{\mu} b_{L}^{i}, \quad \widetilde{O}_{S-P}=\bar{b}_{R}^{i} q_{L}^{j} \bar{q}_{L}^{j} b_{R}^{i}
$$

instead of $T_{V-A}$ and $T_{S-P}$.

The heavy-quark spin symmetry, i.e. the fact that interactions with the spin of the heavy quark decouple as the heavy-quark mass tends to infinity, allows us to derive two relations between the matrix elements of the four-quark operators between $\Lambda_{b}$-baryon states. To find these relations, we note that the following matrix element vanishes in the limit $m_{b} \rightarrow \infty$ :

$$
\frac{1}{2 m_{\Lambda_{b}}}\left\langle\Lambda_{b}\left|\bar{b}^{i} \gamma_{\mu} \gamma_{5} b^{j} \bar{q}_{L}^{k} \gamma^{\mu} q_{L}^{l}\right| \Lambda_{b}\right\rangle=O\left(1 / m_{b}\right) .
$$

The physical argument for this is that, because of the spin symmetry for heavy quarks, the matrix elements for left-handed and right-handed $b$ quarks must be

the same. The above result then follows since $\bar{b} \gamma_{\mu} \gamma_{5} b=\bar{b}_{R} \gamma_{\mu} b_{R}-b_{L} \gamma_{\mu} b_{L}$. A more formal argument can be given using the covariant tensor formalism of the HQET 25, 31 to show that the matrix element in (23) is proportional to

$$
\left\langle 2 \mathbf{S}_{b} \cdot \mathbf{S}_{\text {light }}\right\rangle=S_{\Lambda_{b}}\left(S_{\Lambda_{b}}+1\right)-S_{b}\left(S_{b}+1\right)-S_{\text {light }}\left(S_{\text {light }}+1\right)=0,
$$

since, in the heavy-quark limit, the light degrees of freedom are in a state with total spin zero.

Using the Fierz identity

$$
\bar{b}^{i} \gamma_{\mu} \gamma_{5} b^{j} \bar{q}_{L}^{k} \gamma^{\mu} q_{L}^{l}=-2 \bar{b}_{R}^{i} q_{L}^{l} \bar{q}_{L}^{k} b_{R}^{j}-\bar{b}_{L}^{i} \gamma_{\mu} q_{L}^{l} \bar{q}_{L}^{k} \gamma^{\mu} b_{L}^{j}
$$

we then obtain the relations

$$
\begin{aligned}
& \frac{1}{2 m_{\Lambda_{b}}}\left\langle\Lambda_{b}\left|O_{S-P}\right| \Lambda_{b}\right\rangle=-\frac{1}{2} \frac{1}{2 m_{\Lambda_{b}}}\left\langle\Lambda_{b}\left|O_{V-A}\right| \Lambda_{b}\right\rangle+O\left(1 / m_{b}\right), \\
& \frac{1}{2 m_{\Lambda_{b}}}\left\langle\Lambda_{b}\left|\widetilde{O}_{S-P}\right| \Lambda_{b}\right\rangle=-\frac{1}{2} \frac{1}{2 m_{\Lambda_{b}}}\left\langle\Lambda_{b}\left|\widetilde{O}_{V-A}\right| \Lambda_{b}\right\rangle+O\left(1 / m_{b}\right) .
\end{aligned}
$$

The corrections of order $1 / m_{b}$ to these relations contribute at order $1 / m_{b}^{4}$ in the heavy-quark expansion and so are negligible to the order we work in. This leaves 
us with two independent matrix elements of the operators $O_{V-A}$ and $\widetilde{O}_{V-A}$. The analogue of the factorization approximation in the case of baryons is the valencequark assumption, in which the colour of the quark fields in the operators is identified with the colour of the quarks inside the baryon. Since the colour wave function for a baryon is totally antisymmetric, the matrix elements of $O_{V-A}$ and $\widetilde{O}_{V-A}$ differ in this approximation only by a sign. Hence, we define a parameter $\widetilde{B}$ by

$$
\left\langle\Lambda_{b}\left|\widetilde{O}_{V-A}\right| \Lambda_{b}\right\rangle \equiv-\widetilde{B}\left\langle\Lambda_{b}\left|O_{V-A}\right| \Lambda_{b}\right\rangle,
$$

with $\widetilde{B}=1$ in the valence-quark approximation.

For the baryon matrix element of $O_{V-A}$ itself, our parametrization is guided by the quark model. We write

$$
\frac{1}{2 m_{\Lambda_{b}}}\left\langle\Lambda_{b}\left|O_{V-A}\right| \Lambda_{b}\right\rangle \equiv-\frac{f_{B}^{2} m_{B}}{48} r
$$

where in the quark model $r$ is the ratio of the squares of the wave functions determining the probability to find a light quark at the location of the $b$ quark inside the $\Lambda_{b}$ baryon and the $B$ meson, i.e. [9, 10]

$$
r=\frac{\left|\psi_{b q}^{\Lambda_{b}}(0)\right|^{2}}{\left|\psi_{b \bar{q}}^{B_{q}}(0)\right|^{2}}
$$

Guberina et al. have estimated the ratio $r$ for charm decays and find $r \simeq 0.2$ in the bag model, and $r \simeq 0.5$ in the non-relativistic quark model [8]. This latter estimate has been used in more recent work on beauty decays [6]. A similar result, $r \sim 0.1-0.3$, has been obtained by Colangelo and Fazio using QCD sum rules 32]. Recently, Rosner has criticized the existing quark-model estimates of $r$. Assuming that the wave functions of the $\Lambda_{b}$ and $\Sigma_{b}$ baryons are the same, he argues that the wave-function ratio should be estimated from the ratio of the spin splittings between $\Sigma_{b}$ and $\Sigma_{b}^{*}$ baryons and $B$ and $B^{*}$ mesons [33]. This leads to

$$
r=\frac{4}{3} \frac{m_{\Sigma_{b}^{*}}^{2}-m_{\Sigma_{b}}^{2}}{m_{B^{*}}^{2}-m_{B}^{2}}
$$

If the baryon splitting is taken to be $m_{\Sigma_{b}^{*}}^{2}-m_{\Sigma_{b}}^{2} \simeq m_{\Sigma_{c}^{*}}^{2}-m_{\Sigma_{c}}^{2}=(0.384 \pm$ $0.035) \mathrm{GeV}^{2}$, this leads to $r \simeq 0.9 \pm 0.1$. If, on the other hand, one uses the preliminary result $m_{\Sigma_{b}^{*}}-m_{\Sigma_{b}}=(56 \pm 16) \mathrm{MeV}$ reported by the DELPHI Collaboration [34, one obtains $r \simeq 1.8 \pm 0.5$. We conclude that it is conceivable that $r \sim 1$, i.e. larger than previous estimates.

In terms of these parameters, the matrix element of $\boldsymbol{\Gamma}_{\text {spec }}$ is given by

$$
\frac{1}{2 m_{\Lambda_{b}}}\left\langle\Lambda_{b}\left|\boldsymbol{\Gamma}_{\text {spec }}\right| \Lambda_{b}\right\rangle=\Gamma_{0} \eta_{\text {spec }} \frac{r}{16}\left\{4(1-z)^{2}\left[\left(c_{-}^{2}-c_{+}^{2}\right)+\left(c_{-}^{2}+c_{+}^{2}\right) \widetilde{B}\right]\right.
$$




$$
\begin{gathered}
-\left[(1-z)^{2}(1+z) \cos ^{2} \theta_{c}+\sqrt{1-4 z} \sin ^{2} \theta_{c}\right] \\
\left.\times\left[\left(c_{-}-c_{+}\right)\left(5 c_{+}-c_{-}\right)+\left(c_{-}+c_{+}\right)^{2} \widetilde{B}\right]\right\} .
\end{gathered}
$$

\subsection{Numerical results}

To illustrate the main features of our results, we calculate the coefficients of the hadronic parameters $B_{i}, \varepsilon_{i}$, and $r$ and $\widetilde{B} r$ in the matrix elements (18) and (31) in units of $\Gamma_{0} \eta_{\mathrm{spec}}$. In order to study the dependence on the mass ratio $z=\left(m_{c} / m_{b}\right)^{2}$, we first keep the values of the Wilson coefficients in the effective Lagrangian fixed and vary the mass ratio in the range $z=0.085 \pm 0.015$. This leads to the numbers shown in table 1, where the variation with $z$ is indicated as a change in the last digit(s). Note that for mesons the coefficients of the parameters $B_{i}$ are much smaller than those of the parameters $\varepsilon_{i}$. It is apparent that the results are rather stable with respect to the precise value of $z$. From now on we shall always use the central value $z=0.085$, which is obtained, for instance, for $m_{c}=1.4 \mathrm{GeV}$ and $m_{b}=4.8 \mathrm{GeV}$.

Table 1: Coefficients of the hadronic parameters obtained for $z=0.085 \pm$ 0.015 . The values $c_{+}=0.861$ and $c_{-}=1.349$ are kept fixed.

\begin{tabular}{|c|cccc|cc|}
\hline \hline$H_{b}$ & $B_{1}$ & $B_{2}$ & $\varepsilon_{1}$ & $\varepsilon_{2}$ & $r$ & $\widetilde{B} r$ \\
\hline$B^{-}$ & $-0.28(1)$ & - & $6.43(21)$ & - & & \\
$B_{d}$ & $-0.04(0)$ & $0.05(0)$ & $-2.12(6)$ & $2.39(2)$ & & \\
$B_{s}$ & $-0.03(0)$ & $0.04(0)$ & $-1.83(11)$ & $2.32(5)$ & & \\
$\Lambda_{b}$ & & & & & $0.14(1)$ & $0.26(1)$ \\
\hline \hline
\end{tabular}

As another check on the stability of the results we present in table 2 the coefficients obtained using different "matching" procedures. To this end, we renormalize the Wilson coefficients $c_{+}$and $c_{-}$of the effective Lagrangian at a scale $\mu$ different from $m_{b}$. Thus $c_{+}$and $c_{-}$are modified by replacing $m_{b}$ by $\mu$ in (6). This gives us predictions in terms of operators renormalized at $\mu$, which we then rewrite in terms of those defined at $m_{b}$ by using the evolution equations given in appendix A. Thus, the meaning of the hadronic parameters is the same as before, and the differences between the numerical results can be viewed as an estimate of unknown higher-order perturbative corrections, which we neglect throughout this paper. The coefficients of the parameters $B_{i}$ for mesons, as well as of the parameters $r$ and $\widetilde{B} r$ for the $\Lambda_{b}$ baryon, show a significant scale dependence. To reduce this dependence would require a full next-to-leading order calculation of radiative corrections, which is beyond the scope of this paper. 
Table 2: Coefficients of the hadronic parameters obtained for the matching scales $\mu=m_{b} / 2, m_{b}$ and $2 m_{b}$, with $m_{b}=4.8 \mathrm{GeV}$. The value $z=0.085$ is kept fixed.

\begin{tabular}{|c|c|c|c|c|c|c|c|}
\hline$H_{b}$ & $\mu$ & $B_{1}$ & $B_{2}$ & $\varepsilon_{1}$ & $\varepsilon_{2}$ & $r$ & $\widetilde{B} r$ \\
\hline \multirow{3}{*}{$B^{-}$} & $m_{b} / 2$ & -0.62 & - & 7.26 & - & & \\
\hline & $m_{b}$ & -0.28 & - & 6.43 & - & & \\
\hline & $2 m_{b}$ & +0.02 & - & 5.90 & - & & \\
\hline \multirow{3}{*}{$B_{d}$} & $m_{b} / 2$ & -0.04 & 0.04 & -2.32 & 2.61 & & \\
\hline & $m_{b}$ & -0.04 & 0.05 & -2.12 & 2.39 & & \\
\hline & $2 m_{b}$ & -0.08 & 0.09 & -1.98 & 2.24 & & \\
\hline \multirow{3}{*}{$B_{s}$} & $m_{b} / 2$ & -0.03 & 0.04 & -2.00 & 2.54 & & \\
\hline & $m_{b}$ & -0.03 & 0.04 & -1.83 & 2.32 & & \\
\hline & $2 m_{b}$ & -0.07 & 0.08 & -1.72 & 2.18 & & \\
\hline \multirow{3}{*}{$\Lambda_{b}$} & $m_{b} / 2$ & & & & & 0.21 & 0.30 \\
\hline & $m_{b}$ & & & & & 0.14 & 0.26 \\
\hline & $2 m_{b}$ & & & & & 0.09 & 0.23 \\
\hline
\end{tabular}

\section{Phenomenology of beauty lifetimes}

We shall now discuss the phenomenological implications of our results for the calculation of beauty lifetime ratios. The spectator contributions to the decay widths of $B_{q}$ mesons and of the $\Lambda_{b}$ baryon are described by a set of hadronic parameters: $B_{1,2}$ and $\varepsilon_{1,2}$ for mesons, and $r$ and $\widetilde{B}$ for baryons. The explicit dependence of the decay rates on these quantities is shown in (18) and (31). The numerical values of the coefficients multiplying the hadronic parameters are given in table 2 for three different choices of the matching scale $\mu$. For the numerical analysis we need the value of the parameter $\eta_{\text {spec }}$ defined in (19). We take $f_{B}=200 \mathrm{MeV}$ and $m_{b}=4.8 \mathrm{GeV}$, so that $\eta_{\text {spec }} \simeq 0.30$, and absorb the uncertainty in $\eta_{\text {spec }}$ into the values of the hadronic parameters.

\subsection{Lifetime ratio $\tau\left(B^{-}\right) / \tau\left(B_{d}\right)$}

We start by discussing the lifetime ratio of the charged and neutral $B$ mesons. Because of isospin symmetry, the lifetimes of these states are the same at order $1 / m_{b}^{2}$ in the heavy-quark expansion, and differences arise only from spectator effects. If we write

$$
\frac{\tau\left(B^{-}\right)}{\tau\left(B_{d}\right)}=1+k_{1} B_{1}+k_{2} B_{2}+k_{3} \varepsilon_{1}+k_{4} \varepsilon_{2},
$$

the coefficients $k_{i}$ take the values shown in table 3. The most striking feature of this result is the large imbalance between the coefficients of the parameters $B_{i}$ 
and $\varepsilon_{i}$, which parametrize the matrix elements of colour singlet-singlet and colour octet-octet operators, respectively. With $\varepsilon_{i}$ of order $1 / N_{c}$, it is conceivable that the non-factorizable contributions actually dominate the result. Thus, without a detailed calculation of the parameters $\varepsilon_{i}$ no reliable prediction can be obtained. In this conclusion we disagree with the authors of ref. [6], who use factorization (at a low hadronic scale) to argue that $\tau\left(B^{-}\right) / \tau\left(B_{d}\right)$ must exceed unity by an amount of order $5 \%$. We will return to this in section 4.4 below.

Table 3: Coefficients $k_{i}$ appearing in (32).

\begin{tabular}{|c|cccc|}
\hline \hline$\mu$ & $k_{1}$ & $k_{2}$ & $k_{3}$ & $k_{4}$ \\
\hline$m_{b} / 2$ & +0.044 & 0.003 & -0.735 & 0.201 \\
$m_{b}$ & +0.020 & 0.004 & -0.697 & 0.195 \\
$2 m_{b}$ & -0.008 & 0.007 & -0.665 & 0.189 \\
\hline \hline
\end{tabular}

The experimental value of the lifetime ratio given in (1) can be employed to constrain a certain combination of the parameters:

$$
\varepsilon_{1}=\frac{1}{k_{3}}\left[(0.02 \pm 0.04)-k_{1} B_{1}-k_{2} B_{2}-k_{4} \varepsilon_{2}\right] .
$$

With the values of the coefficients given in table 3, this relation implies

$$
\varepsilon_{1} \simeq 0.3 \varepsilon_{2}+\delta
$$

where we expect $|\delta|<0.1$. This becomes a particularly useful constraint if the parameters $\varepsilon_{i}$ turn out to be large. Below, we shall use (33) to eliminate $\varepsilon_{1}$ from our predictions for spectator effects.

\subsection{Lifetime ratio $\tau\left(B_{s}\right) / \tau\left(B_{d}\right)$}

In the limit where $\mathrm{SU}(3)$-breaking effects are neglected, the spectator contributions to the decay widths of $B_{s}$ and $B_{d}$ mesons are too similar to produce an observable lifetime difference (see table 2). The corresponding contributions to the lifetime ratio $\tau\left(B_{s}\right) / \tau\left(B_{d}\right)$ are of order $10^{-3}$. A precise prediction for $\mathrm{SU}(3)$ breaking effects is difficult to obtain. Allowing for $30 \% \mathrm{SU}(3)$-breaking effects in the matrix elements of the four-quark operators describing the spectator contributions, we estimate that the resulting contributions to the lifetime ratio are of order $1-2 \%$. Contributions of order $1 \%$ (or less) could also arise from SU(3)breaking effects in the matrix elements appearing at order $1 / m_{b}^{2}$ in the expansion (11). Hence, we agree with ref. [6] that

$$
\frac{\tau\left(B_{s}\right)}{\tau\left(B_{d}\right)}=1 \pm O(1 \%) \text {. }
$$




\subsection{Lifetime ratio $\tau\left(\Lambda_{b}\right) / \tau\left(B_{d}\right)$}

As mentioned in the introduction, the low experimental value of the lifetime ratio $\tau\left(\Lambda_{b}\right) / \tau\left(B_{d}\right)$ is the primary motivation for our study. We shall now discuss the structure of spectator contributions to this ratio. It is important that heavy-quark symmetry allows us to reduce the number of hadronic parameters contributing to the decay rate of the $\Lambda_{b}$ baryon from four to two, and that these parameters are almost certainly positive (unless the quark model is completely misleading) and enter the decay rate with the same sign. Thus, unlike in the meson case, the structure of the spectator contributions to the width of the $\Lambda_{b}$ baryon is rather simple, and at least the sign of the effects can be predicted reliably.

For a more detailed discussion, we distinguish between the two cases where one does or does not allow spectator contributions to enhance the theoretical prediction for the semileptonic branching ratio, $B_{\mathrm{SL}}$, of $B$ mesons. As we will discuss below, the theoretical prediction for $B_{\mathrm{SL}}$, which neglects spectator contributions, is slightly larger than the central experimental value. If spectator effects increased the prediction for $B_{\mathrm{SL}}$ further, this discrepancy could become uncomfortably large.

If we do not allow for an increase in the value of the semileptonic branching ratio, the explanation of the low value of $\tau\left(\Lambda_{b}\right) / \tau\left(B_{d}\right)$ must reside entirely in a low value of the $\Lambda_{b}$ lifetime (rather than a large value of the $B$-meson lifetime). This can be seen by writing

$$
\frac{\tau\left(\Lambda_{b}\right)}{\tau\left(B_{d}\right)}=\tau\left(\Lambda_{b}\right)\left(\frac{\tau\left(B^{-}\right)}{\tau\left(B_{d}\right)}\right)^{1 / 2} \frac{1}{\left[\tau\left(B^{-}\right) \tau\left(B_{d}\right)\right]^{1 / 2}}=\frac{\tau\left(\Lambda_{b}\right)}{B_{\mathrm{SL}}}\left(\frac{\tau\left(B^{-}\right)}{\tau\left(B_{d}\right)}\right)^{1 / 2} \Gamma_{\mathrm{SL}}(B)
$$

where $B_{\mathrm{SL}}$ is the average semileptonic branching ratio of $B$ mesons, and $\Gamma_{\mathrm{SL}}(B)$ is the semileptonic width. In the second step we have replaced the geometric mean $\left[\tau\left(B^{-}\right) \tau\left(B_{d}\right)\right]^{1 / 2}$ by the average $B$-meson lifetime, which because of isospin symmetry is correct to order $1 / m_{b}^{6}$ in the heavy-quark expansion. Since there are no spectator contributions to the semileptonic rate $\Gamma_{\mathrm{SL}}(B)$, and since we do not allow an enhancement of the semileptonic branching ratio, in order to obtain a small value for $\tau\left(\Lambda_{b}\right) / \tau\left(B_{d}\right)$ we can increase the width of the $\Lambda_{b}$ baryon and/or decrease (within the experimental errors) the lifetime ratio $\tau\left(B^{-}\right) / \tau\left(B_{d}\right)$. Allowing for a downward fluctuation of this ratio by two standard deviations, i.e. $\tau\left(B^{-}\right) / \tau\left(B_{d}\right)>0.94$, and using the estimate of $1 / m_{b}^{2}$ corrections in (2), we conclude that

$$
\frac{\tau\left(\Lambda_{b}\right)}{\tau\left(B_{d}\right)}>0.97 \times\left[0.98-\frac{\Gamma_{\mathrm{spec}}\left(\Lambda_{b}\right)}{\Gamma\left(\Lambda_{b}\right)}\right]=0.95-\left(d_{1}+d_{2} \widetilde{B}\right) r
$$

where $\Gamma_{\text {spec }}\left(\Lambda_{b}\right)$ is the spectator contribution to the width of the $\Lambda_{b}$ baryon. The values of the coefficients $d_{i}$ are given in table 4 . If we assume that $r$ and $\widetilde{B}$ are of order unity, we find that the spectator contributions yield a reduction of 
the lifetime of the $\Lambda_{b}$ baryon by a few per cent, and that $\tau\left(\Lambda_{b}\right) / \tau\left(B_{d}\right)>0.9$, in contrast with the experimental result given in (1). If, for example, we try to push the theoretical prediction by taking the large value $\widetilde{B}=1.5$ (corresponding to a violation of the valence-quark approximation by $50 \%$ ) and choosing a low matching scale $\mu=m_{b} / 2$, we have to require that $r>r_{\min }$ with $r_{\min }=3.1$, 2.2 and 1.3 for $\tau\left(\Lambda_{b}\right) / \tau\left(B_{d}\right)=0.78,0.83$ and 0.88 (corresponding to the central experimental value and the $1 \sigma$ and $2 \sigma$ fluctuations). Hence, even if we allow for an upward fluctuation of the experimental result by two standard deviations, we need a value of $r$ that is significantly larger than most quark-model predictions (see the discussion in section 3.2). Clearly, a reliable field-theoretic calculation of the parameters $r$ and $\widetilde{B}$ is of great importance to support or rule out such a possibility.

Table 4: Coefficients $d_{i}$ appearing in (37) and (38).

\begin{tabular}{|c|cccc|}
\hline \hline$\mu$ & $d_{1}$ & $d_{2}$ & $d_{3}$ & $d_{4}$ \\
\hline$m_{b} / 2$ & 0.016 & 0.023 & 0.178 & -0.201 \\
$m_{b}$ & 0.012 & 0.021 & 0.173 & -0.195 \\
$2 m_{b}$ & 0.008 & 0.020 & 0.167 & -0.189 \\
\hline \hline
\end{tabular}

On the other hand, the low experimental value of the semileptonic branching ratio may find its explanation in a low renormalization scale (see section 5 below), or it may be caused by the effects of New Physics, such as an enhanced rate for flavour-changing neutral currents of the type $b \rightarrow s g$ [35] 38]. Hence, one may be misled in using the semileptonic branching ratio as a constraint on the size of spectator contributions. Then there is the possibility to decrease the value of $\tau\left(\Lambda_{b}\right) / \tau\left(B_{d}\right)$ by increasing the lifetime of the $B_{d}$ meson, i.e. in (37) we can allow for spectator contributions to the width of the $B_{d}$ meson. From table 2 , it follows that the contributions of the parameters $B_{1}$ and $B_{2}$ are very small (of order $10^{-3}$ ) and can safely be neglected. Thus, we obtain

$$
\frac{\tau\left(\Lambda_{b}\right)}{\tau\left(B_{d}\right)} \simeq 0.98-\left(d_{1}+d_{2} \widetilde{B}\right) r-\left(d_{3} \varepsilon_{1}+d_{4} \varepsilon_{2}\right),
$$

where the coefficients $d_{3}$ and $d_{4}$ are also shown in table 1 . At first sight, it seems that with a positive $\varepsilon_{1}$ and a negative $\varepsilon_{2}$ of order $1 / N_{c}$ one could gain a contribution of about -0.1 , which would take away much of the discrepancy between theory and experiment. However, the experimental result for the lifetime ratio $\tau\left(B^{-}\right) / \tau\left(B_{d}\right)$ imposes a useful constraint. Using (33) to eliminate $\varepsilon_{1}$ from the relation (38), and allowing the parameters $B_{i}$ to take values between 0 and 2 , we find

$$
\frac{\tau\left(\Lambda_{b}\right)}{\tau\left(B_{d}\right)} \simeq 0.98 \pm 0.02+0.15 \varepsilon_{2}-\left(d_{1}+d_{2} \widetilde{B}\right) r>0.88-\left(d_{1}+d_{2} \widetilde{B}\right) r
$$


where in the last step we have assumed that $\left|\varepsilon_{2}\right|<0.5$, which we consider to be a very conservative bound. Even in this extreme case, a significant contribution must still come from the parameters $r$ and $\widetilde{B}$.

In view of the above discussion, the short $\Lambda_{b}$ lifetime remains a potential problem for the heavy-quark theory. If the current experimental value persists, there are two possibilities: either some hadronic matrix elements of four-quark operators are significantly larger than naive expectations based on large- $N_{c}$ counting rules and the quark model, or (local) quark-hadron duality, which is assumed in the calculation of lifetimes, fails in non-leptonic inclusive decays. In the second case, the explanation of the puzzle lies beyond the heavy-quark expansion. Let us, therefore, consider the first possibility and give a numerical example for some possible scenarios. Assume that $\mu=m_{b} / 2$ is an appropriate scale to use in the evaluation of the Wilson coefficients, and that $\widetilde{B}=1.5$. Then, to obtain $\tau\left(\Lambda_{b}\right) / \tau\left(B_{d}\right)=0.8$ without enhancing the prediction for the semileptonic branching ratio requires $r \simeq 3$, i.e. several times larger than quark-model estimates. If, on the other hand, we consider $r=1.5$ as the largest conceivable value, we need $\varepsilon_{2} \simeq-0.5$, corresponding to a rather large matrix element of the colour-octet operator $T_{\mathrm{S}-\mathrm{P}}$. Such a value of $\varepsilon_{2}$ leads to an enhancement of the $B$-meson lifetime, and hence to an enhancement of the semileptonic branching ratio of $B$ mesons, by $\Delta B_{\mathrm{SL}} \simeq 1 \%$. As we will discuss in section 5, this is still tolerable provided yet unknown higher-order corrections confirm the use of a low renormalization scale. Although in both cases some large parameters are needed, we find it important to note that until reliable field-theoretic calculations of the matrix elements of fourquark operators become available, a conventional explanation of the $\Lambda_{b}$-lifetime puzzle cannot be excluded.

\subsection{Relation with the conventional factorization approach}

We now discuss in detail the relation of our approach with previous analyses based on the factorization approximation [6]. This approximation amounts to setting (see appendix A)

$$
B_{i}\left(\mu_{\mathrm{had}}\right)=\left(\frac{\alpha_{s}\left(m_{b}\right)}{\alpha_{s}\left(\mu_{\mathrm{had}}\right)}\right)^{4 / \beta_{0}}, \quad \varepsilon_{i}\left(\mu_{\mathrm{had}}\right)=0,
$$

at some hadronic scale $\mu_{\text {had }} \ll m_{b}$. Here $\beta_{0}$ is the first coefficient of the $\beta$ function. The evolution of the operators from $m_{b}$ to $\mu_{\text {had }}$ is done to leading logarithmic order [10, 27, 28]. For the purpose of our discussion, we fix $\mu_{\text {had }}$ such that $\alpha_{s}\left(\mu_{\text {had }}\right)=0.5$. Then the relation between the hadronic parameters renormalized at the scale $m_{b}$ with those renormalized at $\mu_{\text {had }}$, as given in (A.5), is:

$$
\begin{aligned}
B_{i}\left(m_{b}\right) & \simeq 1.48 B_{i}\left(\mu_{\mathrm{had}}\right)-0.36 \varepsilon_{i}\left(\mu_{\mathrm{had}}\right) \\
\varepsilon_{i}\left(m_{b}\right) & \simeq-0.08 B_{i}\left(\mu_{\mathrm{had}}\right)+1.06 \varepsilon_{i}\left(\mu_{\mathrm{had}}\right) .
\end{aligned}
$$


The theoretical prediction for the lifetime ratio $\tau\left(B^{-}\right) / \tau\left(B_{d}\right)$ in terms of the hadronic parameters renormalized at the scale $\mu_{\text {had }}$ is obtained by combining (41) with the numbers given in table 3. We find

$$
\frac{\tau\left(B^{-}\right)}{\tau\left(B_{d}\right)} \simeq 1+0.08 B_{1}\left(\mu_{\mathrm{had}}\right)-0.01 B_{2}\left(\mu_{\mathrm{had}}\right)-0.75 \varepsilon_{1}\left(\mu_{\mathrm{had}}\right)+0.20 \varepsilon_{2}\left(\mu_{\mathrm{had}}\right) .
$$

The factorization approximation $B_{i}\left(\mu_{\text {had }}\right) \simeq 0.68$ and $\varepsilon_{i}\left(\mu_{\text {had }}\right)=0$ leads to $\tau\left(B^{-}\right) / \tau\left(B_{d}\right) \simeq 1.05$, which is close to the value obtained by Bigi et al. [6]. However, it is evident from (42) that even at a low scale the coefficients of the non-factorizable terms are still much larger than those of the factorizable ones. Hence, it remains true that non-factorizable corrections are potentially important. It would be justified to neglect these contributions only if the parameters $\varepsilon_{i}\left(\mu_{\text {had }}\right)$ were significantly smaller than $10 \%$. However, to the best of our knowledge there is currently no compelling argument to support such a strong restriction.

\section{Semileptonic branching ratio of $B$ mesons}

The semileptonic branching ratio of $B$ mesons has received considerable attention in the past. It is defined as

$$
B_{\mathrm{SL}}=\frac{\Gamma(\bar{B} \rightarrow X e \bar{\nu})}{\sum_{\ell} \Gamma(\bar{B} \rightarrow X \ell \bar{\nu})+\Gamma_{\mathrm{NL}}+\Gamma_{\mathrm{rare}}},
$$

where $\Gamma_{\mathrm{NL}}$ and $\Gamma_{\text {rare }}$ are the inclusive rates for non-leptonic and rare decays, respectively. The status of the experimental results on the semileptonic branching ratio is controversial, as there is a discrepancy between low-energy measurements performed at the $\Upsilon(4 s)$ resonance and high-energy measurements performed at the $Z^{0}$ resonance. The average value at low energies is $B_{\mathrm{SL}}=(10.37 \pm 0.30) \%$ [39], whereas high-energy measurements give $B_{\mathrm{SL}}^{(b)}=(11.11 \pm 0.23) \%$ [40]. The superscript $(b)$ indicates that this value refers not to the $B$ meson, but to a mixture of $b$ hadrons (approximately $40 \% B^{-}, 40 \% \bar{B}_{d}, 12 \% B_{s}$, and $8 \% \Lambda_{b}$ ). Assuming that the corresponding semileptonic width $\Gamma_{\mathrm{SL}}^{(b)}$ is close to that of the $B$ meson, \& we can correct for this and find $B_{\mathrm{SL}}=(\tau(B) / \tau(b)) B_{\mathrm{SL}}^{(b)}=(11.30 \pm$ $0.26) \%$, where $\tau(b)=(1.57 \pm 0.03)$ ps is the average lifetime corresponding to the above mixture of $b$ hadrons [1]. The discrepancy between the low-energy and high-energy measurements of the semileptonic branching ratio is therefore larger than 3 standard deviations. If we take the average and inflate the error to account for this fact, we obtain

$$
B_{\mathrm{SL}}=(10.90 \pm 0.46) \% \text {. }
$$

\footnotetext{
${ }^{2}$ Theoretically, this is expected to be a very good approximation.
} 
In understanding this result, an important aspect is charm counting, i.e. the measurement of the average number $n_{c}$ of charm hadrons produced per $B$ decay. Theoretically, this quantity is given by

$$
n_{c}=1+B\left(\bar{B} \rightarrow X_{c \bar{c} \bar{s}^{\prime}}\right)-B(\bar{B} \rightarrow \text { no charm }),
$$

where $B\left(\bar{B} \rightarrow X_{c \bar{s}^{\prime}}\right)$ is the branching ratio for decays into final states containing two charm quarks, and $B(\bar{B} \rightarrow$ no charm $) \sim 0.02$ [14, 41, 42] is the Standard Model branching ratio for charmless decays. Recently, two new measurements of the average charm content have been performed. The CLEO Collaboration has presented the value $n_{c}=1.16 \pm 0.05$ [39, 43], and the ALEPH Collaboration has reported the result $n_{c}=1.23 \pm 0.07$ [44]. The average is

$$
n_{c}=1.18 \pm 0.04 .
$$

The naive parton model predicts that $B_{\mathrm{SL}} \simeq 15 \%$ and $n_{c} \simeq 1.2$; however, it has been known for some time that perturbative corrections could change these results significantly [14]. With the establishment of the $1 / m_{Q}$ expansion, the non-perturbative corrections to the parton model could be computed, and their effect turned out to be very small. This led Bigi et al. to conclude that values $B_{\mathrm{SL}}<12.5 \%$ cannot be accommodated by theory, thus giving rise to a puzzle referred to as the "baffling semileptonic branching ratio" 15]. Recently, Bagan et al. have completed the calculation of the $O\left(\alpha_{s}\right)$ corrections including the effects of the charm-quark mass [16], finding that they lower the value of $B_{\mathrm{SL}}$ significantly.

The analysis of Bagan et al. has been corrected in an erratum [16]. Here we shall present the results of an independent numerical analysis using the same theoretical input. As the subject is of considerable importance, we shall explain our analysis in detail. The semileptonic branching ratio and $n_{c}$ depend on the pole masses of the heavy quarks, which we allow to vary in the range

$$
m_{b}=(4.8 \pm 0.2) \mathrm{GeV}, \quad m_{b}-m_{c}=(3.40 \pm 0.06) \mathrm{GeV},
$$

corresponding to $0.25<m_{c} / m_{b}<0.33$. Here $m_{b}$ is the pole mass defined to oneloop order in perturbation theory. The difference $m_{b}-m_{c}$ is free of renormalon ambiguities and can be determined from spectroscopy (see, e.g., ref. [7]). Bagan et al. have also considered the theoretical predictions in a scheme where the quark masses are renormalized at a scale $\mu$ in the $\overline{\mathrm{MS}}$ scheme. We discuss this scheme in appendix B. At order $1 / m_{b}^{2}$ in the heavy-quark expansion, non-perturbative effects are described by the single parameter $\mu_{G}^{2}(B)$ defined in (9); the dependence on the parameter $\mu_{\pi}^{2}(B)$ is the same for all inclusive decay rates and cancels out in $B_{\mathrm{SL}}$ and $n_{c}$. Moreover, the results depend on the scale $\mu$ used to renormalize the coupling constant $\alpha_{s}(\mu)$ and the Wilson coefficients $c_{ \pm}(\mu)$ entering the nonleptonic decay rate. They also depend on the value of the QCD scale parameter 
$\Lambda_{\mathrm{QCD}}$, which we fix taking $\alpha_{s}\left(m_{Z}\right)=0.117 \pm 0.004$. The corresponding uncertainty is smaller than that due to the variation of the mass parameters and is added in quadrature. For the two choices $\mu=m_{b}$ and $\mu=m_{b} / 2$, we obtain

$$
\begin{aligned}
B_{\mathrm{SL}} & = \begin{cases}12.0 \pm 1.0 \% ; & \mu=m_{b}, \\
10.9 \pm 1.0 \% ; & \mu=m_{b} / 2,\end{cases} \\
n_{c} & = \begin{cases}1.20 \mp 0.06 ; & \mu=m_{b}, \\
1.21 \mp 0.06 ; & \mu=m_{b} / 2 .\end{cases}
\end{aligned}
$$

The errors in the two quantities are anticorrelated. Notice that the semileptonic branching ratio has a much stronger scale dependence than $n_{c}$. This is illustrated in figure 2, where we show the two quantities as a function of $\mu$. By choosing a low renormalization scale, values $B_{\mathrm{SL}}<12.5 \%$ can easily be accommodated. The experimental data prefer a scale $\mu / m_{b} \sim 0.3-0.5$, which is indeed not unnatural. Using the BLM scale-setting method [45], Luke et al. have estimated that $\mu \simeq$ $0.3 m_{b}$ is an appropriate scale in this case [46].
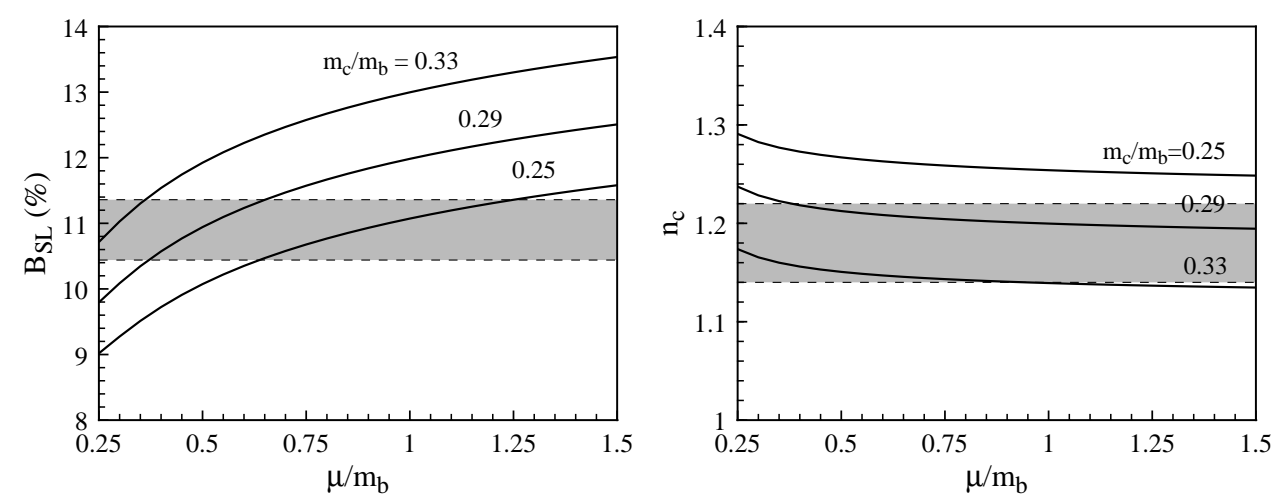

Figure 2: Scale dependence of the theoretical predictions for the semileptonic branching ratio and $n_{c}$. The bands show the average experimental values.

The combined theoretical predictions for the semileptonic branching ratio and charm counting are shown in figure 5 . They are compared with the experimental results obtained at the $\Upsilon(4 s)$ and at the $Z^{0}$ resonance. It was argued that the combination of a low semileptonic branching ratio and a low value of $n_{c}$ would constitute a potential problem for the Standard Model [42]. However, with the new experimental and theoretical numbers, only for the low-energy measurements a small discrepancy remains between theory and experiment. Note that, using (45), our results for $n_{c}$ can be used to obtain predictions for the branching ratio $B\left(\bar{B} \rightarrow X_{c \bar{c} s^{\prime}}\right)$, which is accessible to a direct experimental determination. Our prediction of $(22 \pm 6) \%$ for this branching ratio agrees well with the preliminary result of the CLEO Collaboration: $B\left(\bar{B} \rightarrow X_{c \bar{c} s^{\prime}}\right)=(23.9 \pm 3.8) \%$ [47].

Having discussed the status of the theoretical predictions obtained to order $1 / m_{b}^{2}$ in the heavy-quark expansion, we now investigate the spectator contributions to the semileptonic branching ratio and $n_{c}$. This extends, in the context 


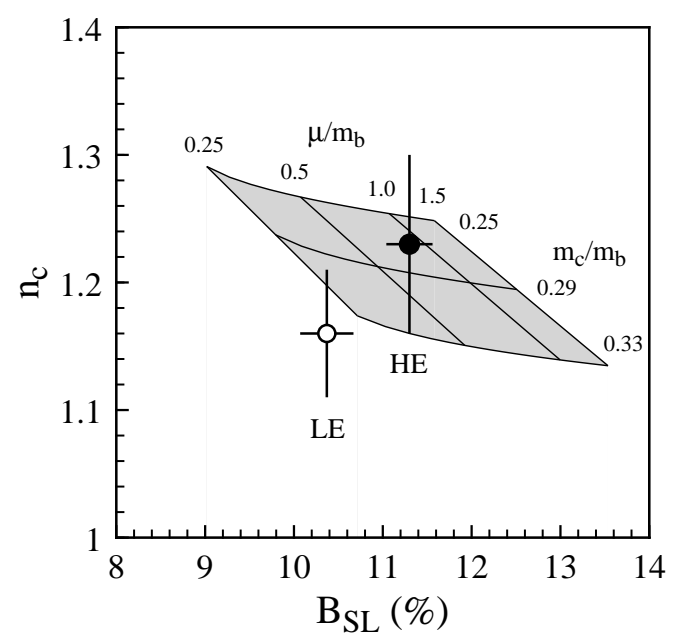

Figure 3: Combined theoretical predictions for the semileptonic branching ratio and charm counting as a function of the quark-mass ratio $m_{c} / m_{b}$ and the renormalization scale $\mu$. The data points show the average experimental values for $B_{\mathrm{SL}}$ and $n_{c}$ obtained in low-energy (LE) and high-energy (HE) measurements.

of the heavy-quark expansion, the phenomenological study presented in ref. [14]. We consider the average of $B_{\mathrm{SL}}$ and $n_{c}$ for $B^{-}$and $B_{d}$ mesons, [] and write for the spectator contributions to these quantities

$$
\begin{aligned}
\Delta B_{\mathrm{SL}, \mathrm{spec}} & =b_{1} B_{1}+b_{2} B_{2}+b_{3} \varepsilon_{1}+b_{4} \varepsilon_{2}, \\
\Delta n_{c, \mathrm{spec}} & =n_{1} B_{1}+n_{2} B_{2}+n_{3} \varepsilon_{1}+n_{4} \varepsilon_{2} .
\end{aligned}
$$

The coefficients $b_{i}$ and $n_{i}$ are given in table 5. If, as previously, we eliminate $\varepsilon_{1}$ from these equations using the constraint (B3) imposed by the measurement of $\tau\left(B^{-}\right) / \tau\left(B_{d}\right)$, and we allow that the parameters $B_{i}$ take values in the range 0 to 2 , we obtain

$$
\begin{aligned}
\Delta B_{\mathrm{SL}, \mathrm{spec}} & \simeq\left(-2.1 \varepsilon_{2}+0.2 \pm 0.3\right) \%, \\
\Delta n_{c, \mathrm{spec}} & \simeq(1.2 \pm 0.1) \Delta B_{\mathrm{SL}, \mathrm{spec}} .
\end{aligned}
$$

For reasonable value of $\varepsilon_{2}$, we expect a contribution to the semileptonic branching ratio of order $1 \%$ or less, and a negligible effect on $n_{c}$. However, without a detailed calculation of the hadronic parameters we cannot obtain a quantitative prediction of the spectator contributions. Nevertheless, we find it interesting that there is at least a potential to change, and in particular to lower, the value of $B_{\mathrm{SL}}$ by 0.5-1\%. To achieve such a decrease requires that the hadronic parameter $\varepsilon_{2}$, which parametrizes the matrix element of the colour octet-octet operator $T_{S-P}^{q}$

\footnotetext{
${ }^{3}$ These are approximately the quantities measured experimentally. However, measurements at $\mathrm{LEP}$ receive a contamination from $B_{s}$ and $b$-baryon decays.
} 
in (13), is positive and of order 0.3-0.5. It will be interesting to see if future calculations of this parameter will confirm or rule out this scenario.

Table 5: Coefficients $b_{i}$ and $n_{i}$ (in \%) appearing in (49).

\begin{tabular}{|c|cccc|}
\hline \hline$\mu$ & $b_{1}$ & $b_{2}$ & $b_{3}$ & $b_{4}$ \\
\hline$m_{b} / 2$ & 0.35 & -0.02 & -2.60 & -1.37 \\
$m_{b}$ & 0.19 & -0.03 & -2.56 & -1.42 \\
$2 m_{b}$ & 0.03 & -0.05 & -2.49 & -1.42 \\
\hline \hline$\mu$ & $n_{1}$ & $n_{2}$ & $n_{3}$ & $n_{4}$ \\
\hline$m_{b} / 2$ & 0.50 & -0.03 & -4.17 & -1.54 \\
$m_{b}$ & 0.25 & -0.03 & -3.79 & -1.44 \\
$2 m_{b}$ & 0.03 & -0.05 & -3.51 & -1.36 \\
\hline \hline
\end{tabular}

\section{Conclusions}

In this paper, we have studied spectator effects in inclusive decays of beauty hadrons. Although these effects are suppressed by three powers of $\Lambda_{\mathrm{QCD}} / m_{b}$ in the heavy-quark expansion, they cannot be neglected because of the large phasespace factor for two-body scattering. The contributions of spectator effects to inclusive decay rates are given by the hadronic matrix elements of the four local operators in (13). For mesons, we have expressed these matrix elements in terms of the hadronic parameters $B_{1,2}$ and $\varepsilon_{1,2}$ defined in (14). For the $\Lambda_{b}$ baryon, heavyquark symmetry reduces the number of independent matrix elements from four to two, which we parametrize by $r$ and $\widetilde{B}$ as defined in (27) and (28). Although our parametrization is motivated by commonly made simplifications, such as the vacuum insertion and the valence-quark approximations, we stress that it is introduced without any loss of generality. For a complete understanding of spectator effects, it will be necessary to evaluate these parameters non-perturbatively, e.g. in lattice simulations.

We find that in predictions for lifetimes and the semileptonic branching ratio of $B$ mesons, the coefficients of the colour octet-octet non-factorizable operators are much larger than those for the colour singlet-singlet factorizable operators. Thus the contributions from the non-factorizable operators cannot be neglected, even though their matrix elements are suppressed in the large- $N_{c}$ limit.

The ratio $\tau\left(B^{-}\right) / \tau\left(B_{d}\right)$ is particularly sensitive to non-factorizable contributions [see (32) and table 3], making it difficult to predict this quantity with a

\footnotetext{
${ }^{4}$ We recall, however, that according to (39) a positive value of $\varepsilon_{2}$ increases the theoretical prediction for the lifetime ratio $\tau\left(\Lambda_{b}\right) / \tau\left(B_{d}\right)$.
} 
precision of better than about $10 \%$. For example, assuming that the magnitudes of the parameters $\varepsilon_{1}$ and $\varepsilon_{2}$ are smaller than 0.1 or 0.2 , we find that the predictions for this lifetime ratio lie in the ranges $0.93-1.11$ and $0.84-1.20$, respectively.f However, in our opinion, even if the experimental result had been outside these ranges, the most likely explanation would have been that the $\varepsilon_{i}$ parameters are larger, rather than a failure of the heavy-quark expansion. The experimental measurement of $\tau\left(B^{-}\right) / \tau\left(B_{d}\right)$ imposes the constraint (33) upon the parameters, which allows us to eliminate $\varepsilon_{1}$ in other relations. On the other hand, within the heavy-quark expansion there is only room for a very small deviation of the ratio $\tau\left(B_{s}\right) / \tau\left(B_{d}\right)$ from unity due to $\mathrm{SU}(3)$-breaking effects. We estimate these effects to be of order $1 \%$.

Understanding the low experimental value of the lifetime ratio $\tau\left(\Lambda_{b}\right) / \tau\left(B_{d}\right)$ remains a potential problem for the heavy-quark theory. If the current experimental value persists, there are two possibilities: either some hadronic matrix elements of four-quark operators are significantly larger than naive expectations based on large- $N_{c}$ counting rules and the quark model, or (local) quark-hadron duality fails in non-leptonic inclusive decays. In the second case, the explanation of the puzzle lies beyond the heavy-quark expansion. In the first case, it is most likely that the baryonic parameter $r$ is much larger than most expectations based on quark-model estimates. It will be interesting to see whether future, field-theoretic calculations will yield values of $r$ which are sufficiently large. Until such calculations become available, a conventional explanation of the $\Lambda_{b}$-lifetime puzzle cannot be excluded.

Finally, we have performed an analysis of the semileptonic branching ratio of the $B$ meson $\left(B_{\mathrm{SL}}\right)$ and of the average number of charmed particles produced per decay $\left(n_{c}\right)$. Our results are summarized in figures 2 and 3 . There is a significant dependence on the predictions for the semileptonic branching ratio on the renormalization scale $\mu$, which is a manifestation of our ignorance of higher-order perturbative corrections. The results for $n_{c}$, on the other hand, are almost independent of $\mu$. This scale dependence weakens considerably the anticorrelation in the theoretically allowed values for $B_{\mathrm{SL}}$ and $n_{c}$ observed in ref. [42. In our view, given the theoretical uncertainties and the disagreement between the experimental values for the semileptonic branching ratio obtained in low- and high-energy measurements, there is at present no discrepancy between theory and experiment for $B_{\mathrm{SL}}$ and $n_{c}$. We have also studied the contributions of spectator effects for these quantities and find that they are negligible for $n_{c}$, whereas they can potentially change the prediction for $B_{\mathrm{SL}}$ by up to about $1 \%$.

Note added: After completing this work we became aware of a paper by I.I. Bigi (preprint UND-HEP-96-BIG01, June 1996 hep-ph/9606405), who dis-

\footnotetext{
${ }^{5}$ Alternatively, if we assume that the magnitudes of $\varepsilon_{1}\left(\mu_{\text {had }}\right)$ and $\varepsilon_{2}\left(\mu_{\text {had }}\right)$ renormalized at a hadronic scale are less than 0.1 and 0.2 , then, using (42), we find that the corresponding predictions lie in the ranges $0.96-1.14$ and $0.87-1.23$.
} 
cusses theoretical predictions for beauty lifetimes, making strong claims concerning the theoretical predictions for the lifetime ratio $\tau\left(B^{-}\right) / \tau\left(B_{d}\right)$. In view of our discussion in section 4.4, we must disagree with some statements made in this paper.

Acknowledgements: We thank Patricia Ball, Jon Rosner, Berthold Stech and Kolia Uraltsev for helpful discussions. C.T.S. acknowledges the Particle Physics and Astronomy Research Council for its support through the award of a Senior Fellowship. 


\section{Appendix A: Renormalization-group evolution}

The four-quark operators appearing in the heavy-quark expansion are conventionally renormalized at the scale $\mu=m_{b}$. However, one may use the renormalizationgroup to rewrite them in terms of operators renormalized at a scale $\mu \neq m_{b}$. The renormalization-group evolution is determined by the anomalous dimensions of the four-quark operators in the HQET, where the $b$ quark is treated as static quark [25]. In the literature, this evolution is sometimes referred to as "hybrid renormalization" [10, 27, 28].

We find that the operators $O_{V-A}^{q}$ and $T_{V-A}^{q}$, and similarly $O_{S-P}^{q}$ and $T_{S-P}^{q}$, mix under renormalization. At one-loop order, the mixing within each pair $(O, T)$ is governed by the anomalous dimension matrix

$$
\hat{\gamma}=\frac{3 \alpha_{s}}{2 \pi}\left(\begin{array}{cc}
C_{F} & 1 \\
-\frac{C_{F}}{2 N_{c}} & \frac{1}{2 N_{c}}
\end{array}\right),
$$

which has eigenvalues 0 and $3 N_{c}$. Here $N_{c}$ is the number of colours, and $C_{F}=$ $\left(N_{c}^{2}-1\right) / 2 N_{c}$ is the eigenvalue of the quadratic Casimir operator in the fundamental representation. The operators defined at the scale $m_{b}$ can be rewritten in terms of those defined at a scale $\mu \neq m_{b}$. In leading logarithmic approximation, the result is

$$
\begin{aligned}
& O\left(m_{b}\right)=\left[1+\frac{2 C_{F}}{N_{c}}(\kappa-1)\right] O(\mu)-\frac{2}{N_{c}}(\kappa-1) T(\mu), \\
& T\left(m_{b}\right)=\left[1+\frac{1}{N_{c}^{2}}(\kappa-1)\right] T(\mu)-\frac{C_{F}}{N_{c}^{2}}(\kappa-1) O(\mu),
\end{aligned}
$$

where

$$
\kappa=\left(\frac{\alpha_{s}(\mu)}{\alpha_{s}\left(m_{b}\right)}\right)^{3 N_{c} / 2 \beta_{0}},
$$

and $\beta_{0}=\frac{11}{3} N_{c}-\frac{2}{3} n_{f}$ is the first coefficient of the $\beta$-function $\left(n_{f}=3\right.$ is the number of light quark flavours).

Given the evolution equations (A.2) for the four-quark operators, it is immediate to derive the corresponding equations for the hadronic parameters defined in $(14),(27)$ and (28). We obtain:

$$
\begin{aligned}
B_{i}\left(m_{b}\right) & =\left[1+\frac{2 C_{F}}{N_{c}}(\kappa-1)\right] B_{i}(\mu)-\frac{2}{N_{c}}(\kappa-1) \varepsilon_{i}(\mu), \\
\varepsilon_{i}\left(m_{b}\right) & =\left[1+\frac{1}{N_{c}^{2}}(\kappa-1)\right] \varepsilon_{i}(\mu)-\frac{C_{F}}{N_{c}^{2}}(\kappa-1) B_{i}(\mu), \\
r\left(m_{b}\right) & =\kappa r(\mu)+\frac{1}{N_{c}}(\kappa-1) \widetilde{B}(\mu) r(\mu), \\
\widetilde{B}\left(m_{b}\right) r\left(m_{b}\right) & =\widetilde{B}(\mu) r(\mu) .
\end{aligned}
$$


Of course, introducing parameters renormalized at a scale $\mu \neq m_{b}$ would simply amount to a reparametrization of the results and as such is not very illuminating. However, the evolution equations are used in section 3 to study the sensitivity of our results to unknown higher-order corrections.

As an illustration, we study the evolution from $\mu=m_{b}$ down to a typical hadronic scale $\mu_{\text {had }} \ll m_{b}$, which we choose such that $\alpha_{s}\left(\mu_{\text {had }}\right)=0.5$ (corresponding to $\left.\mu_{\text {had }} \sim 0.67 \mathrm{GeV}\right)$. We find

$$
\begin{aligned}
B_{i}\left(m_{b}\right) & \simeq 1.48 B_{i}\left(\mu_{\mathrm{had}}\right)-0.36 \varepsilon_{i}\left(\mu_{\mathrm{had}}\right) \\
\varepsilon_{i}\left(m_{b}\right) & \simeq 1.06 \varepsilon_{i}\left(\mu_{\mathrm{had}}\right)-0.08 B_{i}\left(\mu_{\mathrm{had}}\right) \\
r\left(m_{b}\right) & \simeq\left[1.54+0.18 \widetilde{B}\left(\mu_{\mathrm{had}}\right)\right] r\left(\mu_{\mathrm{had}}\right), \\
\widetilde{B}\left(m_{b}\right) & \simeq \frac{\widetilde{B}\left(\mu_{\mathrm{had}}\right)}{1.54+0.18 \widetilde{B}\left(\mu_{\mathrm{had}}\right)}
\end{aligned}
$$

indicating that renormalization effects can be quite significant. If one assumes that the matrix elements renormalized at the scale $\mu_{\text {had }}$ can be estimated using the vacuum insertion hypothesis for mesons and the valence-quark approximation for baryons, then

$$
B_{i}\left(\mu_{\mathrm{had}}\right) \simeq \frac{f_{B}^{2}\left(\mu_{\mathrm{had}}\right)}{f_{B}^{2}\left(m_{b}\right)}=\kappa^{-8 / 9} \simeq 0.68, \quad \varepsilon_{i}\left(\mu_{\mathrm{had}}\right) \simeq 0, \quad \widetilde{B}\left(\mu_{\mathrm{had}}\right) \simeq 1
$$

where the factor $\kappa^{-8 / 9}$ in the first equation arises from the anomalous dimension of the axial current in the HQET [27, 28]. Using (A.4), we then find that the parameters defined at a renormalization scale $m_{b}$ (as used throughout this paper) would be $B_{1}\left(m_{b}\right)=B_{2}\left(m_{b}\right) \simeq 1.01, \varepsilon_{1}\left(m_{b}\right)=\varepsilon_{2}\left(m_{b}\right) \simeq-0.05, r\left(m_{b}\right) / r\left(\mu_{\text {had }}\right) \simeq$ 1.72 , and $\widetilde{B}\left(m_{b}\right) \simeq 0.58$. Thus, for mesons the violations of the factorization approximation induced by the evolution from $\mu_{\text {had }}$ up to $m_{b}$ remain small, i.e. we find $B_{i}\left(m_{b}\right) \simeq 1$ and $\varepsilon_{i}\left(m_{b}\right) \simeq 0$. We stress, however, that we do not want to suggest that the choice of parameters in (A.6) is actually physical.

For mesons, the large- $N_{c}$ counting rules [48, 49] imply that the parameters $\varepsilon_{i}$ are of order $1 / N_{c}$, whereas the parameters $B_{i}$ are of order unity. These results are respected by the evolution equations (A.4), which in the large- $N_{c}$ limit take the form

$$
\begin{aligned}
& B_{i}\left(m_{b}\right)=\kappa_{\infty} B_{i}(\mu)+O\left(1 / N_{c}\right), \\
& \varepsilon_{i}\left(m_{b}\right)=\varepsilon_{i}(\mu)-\frac{1}{2 N_{c}}\left(\kappa_{\infty}-1\right) B_{i}(\mu)+O\left(1 / N_{c}^{2}\right),
\end{aligned}
$$

where $\kappa_{\infty}=\left[\alpha_{s}(\mu) / \alpha_{s}\left(m_{b}\right)\right]^{9 / 22}$. Under a change of the renormalization scale, the parameters $\varepsilon_{i}$ stay of order $1 / N_{c}$, whereas the parameters $B_{i}$ change by a factor of order unity. 


\section{Appendix B: $\quad B_{\mathrm{SL}}$ and $n_{c}$ in the $\overline{\mathrm{MS}}$ scheme}

The semileptonic branching ratio and $n_{c}$ can also be calculated using running quark masses renormalized in the $\overline{\mathrm{MS}}$ scheme rather than pole masses. To compare the results in such a scheme to those presented in our work, we have to relate the ratio of the pole masses to the ratio of the running masses. There is some freedom in how to do this translation. Since in the expressions for the (partial) inclusive decay rates radiative corrections are included to order $\alpha_{s}(\mu)$ only, it is consistent to work with the one-loop relation

$$
\frac{m_{c}}{m_{b}}=\frac{\bar{m}_{c}(\mu)}{\bar{m}_{b}(\mu)}\left(1-\frac{2 \alpha_{s}(\mu)}{\pi} \ln \frac{\bar{m}_{c}(\mu)}{\bar{m}_{b}(\mu)}\right) .
$$

We shall refer to this choice as scheme $\overline{\mathrm{MS}} 1$. Alternatively, one may prefer to resum the leading and next-to-leading logarithms to this relation, which leads to

$$
\frac{\bar{m}_{c}(\mu)}{\bar{m}_{b}(\mu)}=\frac{m_{c}}{m_{b}}\left(\frac{\alpha_{s}\left(m_{c}\right)}{\alpha_{s}\left(m_{b}\right)}\right)^{\gamma_{0} / 2 \beta_{0}}\left\{1-\frac{\alpha_{s}\left(m_{c}\right)-\alpha_{s}\left(m_{b}\right)}{\pi}\left(\frac{\gamma_{1} \beta_{0}-\gamma_{0} \beta_{1}}{8 \beta_{0}^{2}}-\frac{4}{3}\right)\right\},
$$

where $\gamma_{0}$ and $\gamma_{1}$ are the one- and two-loop coefficients of the anomalous dimension of the running quark mass, and $\beta_{0}$ and $\beta_{1}$ are the coefficients of the $\beta$-function. We shall call this scheme $\overline{\mathrm{MS}} 2$; it has been adopted in the work of Bagan et al. [16].

Our results for these two versions of the $\overline{\mathrm{MS}}$ scheme are:

$$
\begin{aligned}
B_{\mathrm{SL}}(\overline{\mathrm{MS}} 1) & = \begin{cases}11.6 \pm 0.9 \% ; & \mu=m_{b}, \\
10.7 \pm 0.9 \% ; & \mu=m_{b} / 2,\end{cases} \\
n_{c}(\overline{\mathrm{MS}} 1) & = \begin{cases}1.20 \mp 0.06 ; & \mu=m_{b}, \\
1.20 \mp 0.06 ; & \mu=m_{b} / 2,\end{cases} \\
B_{\mathrm{SL}}(\overline{\mathrm{MS}} 2) & = \begin{cases}10.9 \pm 0.9 \% ; & \mu=m_{b}, \\
10.3 \pm 0.9 \% ; & \mu=m_{b} / 2,\end{cases} \\
n_{c}(\overline{\mathrm{MS}} 2) & = \begin{cases}1.25 \mp 0.05 ; & \mu=m_{b}, \\
1.24 \mp 0.06 ; & \mu=m_{b} / 2,\end{cases}
\end{aligned}
$$

and the combined predictions for $B_{\mathrm{SL}}$ and $n_{c}$ are shown in figure 4 . Contrary to the case of the on-shell scheme, the calculations in the $\overline{\mathrm{MS}}$ scheme become unstable for low values of the renormalization scale. For this reason, we only present result for $\mu \geq m_{b} / 2$. The results obtained in the scheme $\overline{\mathrm{MS}} 1$ are close to those obtained in the on-shell scheme and presented in section 5. In the scheme $\overline{\mathrm{MS}} 2$, on the other hand, we find lower values for $B_{\mathrm{SL}}$ and higher values for $n_{c}$. We note that our results for this scheme do not coincide with the numbers presented in the erratum of ref. [16]; in particular, we do not find the large values of $n_{c}$ reported there. The numerical differences are mainly due to the fact that Bagan et al. use lower values for the charm-quark mass (they use $m_{c}=1.33 \mathrm{GeV}$ for 
the central value of the pole mass rather than $1.4 \mathrm{GeV}$ ), and that they multiply each partial decay rate by the numerical factor

$$
\left(\frac{m_{b}}{\bar{m}_{b}(\mu)}\right)^{5}=1+\frac{\alpha_{s}(\mu)}{\pi}\left(\frac{20}{3}-5 \ln \frac{m_{b}^{2}}{\mu^{2}}\right)+\ldots
$$

which we omit since it cancels trivially in the dimensionless quantities $B_{\mathrm{SL}}$ and $n_{c}$. Note that, in particular, this factor is responsible for the large apparent scale dependence of the results presented in ref. [16]. Another difference is that we include in the calculation an estimate of the contribution from charmless decays, using $B_{\text {no charm }}=(2 \pm 1) \%$ [14, 41, 42] and $\Gamma_{\mathrm{SL}}^{b \rightarrow u} / \Gamma_{\mathrm{SL}}^{b \rightarrow c} \simeq 1 \%$. This lowers $B_{\mathrm{SL}}$ by a factor 0.99 and $n_{c}$ by a factor 0.98 .

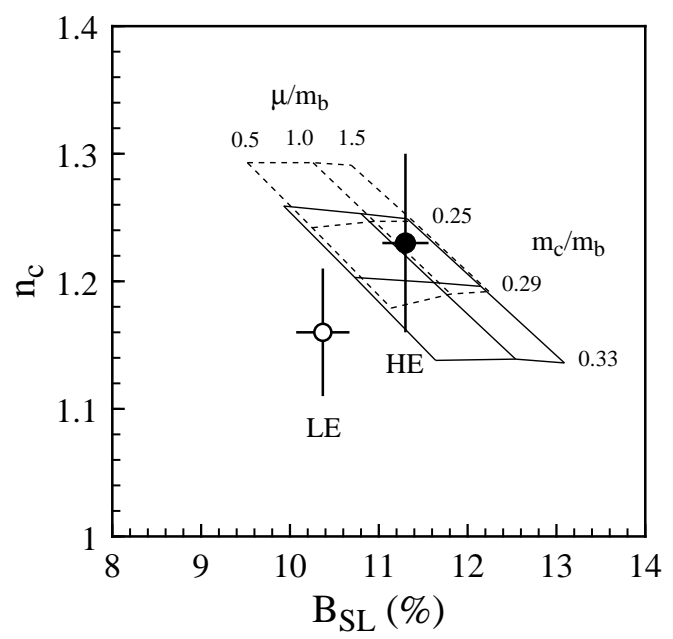

Figure 4: Combined theoretical predictions for the semileptonic branching ratio and charm counting as a function of the quark-mass ratio $m_{c} / m_{b}$ and the renormalization scale $\mu$. The solid lines refer to the scheme $\overline{\mathrm{MS}} 1$, the dashed ones to $\overline{\mathrm{MS}} 2$.

It may be argued that the apparent large scheme dependence of the results for the semileptonic branching ratio and $n_{c}$ prevent a reliable theoretical prediction. However, as we have shown above the main reason is that the numerical value of the quark mass ratio $m_{c} / m_{b}$ can be quite different in different schemes $\left(\bar{m}_{c}(\mu) / \bar{m}_{b}(\mu) \simeq 0.8 m_{c} / m_{b}\right.$ in the scheme $\overline{\mathrm{MS}} 1$, and $0.7 m_{c} / m_{b}$ in $\left.\overline{\mathrm{MS}} 2\right)$. Since the dependence of $B_{\mathrm{SL}}$ and $n_{c}$ on the quark-mass ratio comes simply from phase space (and is particularly strong for the channel $b \rightarrow c \bar{c} s$ ), we feel that the onshell scheme is more adequate for performing the calculation. In other words, we expect that in the $\overline{\mathrm{MS}}$ scheme one would encounter larger higher-order corrections, once the calculation is pushed to order $\alpha_{s}^{2}$ and higher. 


\section{References}

[1] I.J. Kroll, to appear in: Proceedings of the 17th International Symposium on Lepton Photon Interactions (LP95), Beijing, P.R. China, August 1995. The quoted value of $\tau\left(\Lambda_{b}\right) / \tau\left(B_{d}\right)$ includes the new preliminary result of $0.85 \pm$ $0.10 \pm 0.05$ reported in: G. Apollinari (CDF Collaboration), presented at the Aspen Winter Conference on Particle Physics, Aspen, Colorado, January 1996.

[2] J. Chay, H. Georgi and B. Grinstein, Phys. Lett. B 247, 399 (1990).

[3] I.I. Bigi, N.G. Uraltsev and A.I. Vainshtein, Phys. Lett. B 293, 430 (1992) [E: 297, 477 (1993)];

I.I. Bigi, M.A. Shifman, N.G. Uraltsev and A.I. Vainshtein, Phys. Rev. Lett. 71, 496 (1993);

I.I. Bigi et al., in: Proceedings of the Annual Meeting of the Division of Particles and Fields of the APS, Batavia, Illinois, 1992, edited by C. Albright et al. (World Scientific, Singapore, 1993), p. 610.

[4] A.V. Manohar and M.B. Wise, Phys. Rev. D 49, 1310 (1994).

[5] B. Blok, L. Koyrakh, M.A. Shifman and A.I. Vainshtein, Phys. Rev. D 49, 3356 (1994) [E: 50, 3572 (1994)].

[6] B. Blok and M. Shifman, in: Proceedings of the 3rd Workshop on the Tau-Charm Factory, Marbella, Spain, June 1993, edited by J. Kirkby and R. Kirkby (Editions Frontieres, 1994);

I.I. Bigi et al., in: B Decays, edited by S. Stone, Second Edition (World Scientific, Singapore, 1994), p. 134;

I.I. Bigi, preprint UND-HEP-95-BIG02 (1995) hep-ph/9508408].

[7] M. Neubert, preprint CERN-TH/95-307 (1995) [hep-ph/9511409, to appear in: Proceedings of the 17th International Symposium on Lepton Photon Interactions (LP95), Beijing, P.R. China, August 1995; Int. J. Mod. Phys. A 11, 4173 (1996).

[8] B. Guberina, S. Nussinov, R. Peccei and R. Rückl, Phys. Lett. B 89, 111 (1979).

[9] N. Bilic, B. Guberina and J. Trampetic, Nucl. Phys. B 248, 261 (1984);

B. Guberina, R. Rückl and J. Trampetic, Z. Phys. C 33, 297 (1986).

[10] M.A. Shifman and M.B. Voloshin, Sov. J. Nucl. Phys. 41, 120 (1985); JETP 64, 698 (1986).

[11] M.A. Shifman, A.I. Vainshtein and V.I. Zakharov, Nucl. Phys. B 147, 385 and 448 (1979). 
[12] J.L. Cortes and J. Sanchez-Guillen, Phys. Rev. D 24, 2982 (1981).

[13] J.L. Cortes, X.Y. Pham and A. Tounsi, Phys. Rev. D 25, 188 (1982).

[14] G. Altarelli and S. Petrarca, Phys. Lett. B 261, 303 (1991).

[15] I. Bigi, B. Blok, M.A. Shifman and A. Vainshtein, Phys. Lett. B 323, 408 (1994).

[16] E. Bagan, P. Ball, V.M. Braun and P. Gosdzinsky, Nucl. Phys. B 432, 3 (1994); Phys. Lett. B 342, 362 (1995) [E: 374, 363 (1996)];

E. Bagan, P. Ball, B. Fiol and P. Gosdzinsky, Phys. Lett. B 351, 546 (1995).

[17] E.C. Poggio, H.R. Quinn and S. Weinberg, Phys. Rev. D 13, 1958 (1976).

[18] G. Altarelli, G. Martinelli, S. Petrarca and F. Rapuano, preprint CERNTH/96-77 (1996) hep-ph/9604202.

[19] G. Altarelli and L. Maiani, Phys. Lett. B 52, 351 (1974).

[20] M.K. Gaillard and B.W. Lee, Phys. Rev. Lett. 33, 108 (1974).

[21] F.G. Gilman and M.B. Wise, Phys. Rev. D 20, 2392 (1979).

[22] Q. Hokim and X.Y. Pham, Phys. Lett. B 122, 297 (1989).

[23] Y. Nir, Phys. Lett. B 221, 184 (1989).

[24] A.F. Falk, Z. Ligeti, M. Neubert and Y. Nir, Phys. Lett. B 326, 145 (1994).

[25] For a review, see: M. Neubert, Phys. Rep. 245, 259 (1994).

[26] This value is obtained by averaging the result $m_{\Lambda_{b}}=(5639 \pm 15) \mathrm{MeV}$ quoted in ref. [1] with the new preliminary value $m_{\Lambda_{b}}=(5623 \pm 5 \pm 4) \mathrm{MeV}$ reported by the CDF Collaboration in: G. Apollinari (CDF Collaboration), presented at the Aspen Winter Conference on Particle Physics, Aspen, Colorado, January 1996 .

[27] M.A. Shifman and M.B. Voloshin, Sov. J. Nucl. Phys. 45, 292 (1987).

[28] H.D. Politzer and M.B. Wise, Phys. Lett. B 206, 681 (1988); 208, 504 (1988).

[29] I.I. Bigi and N.G. Uraltsev, Nucl. Phys. B 423, 33 (1994); Z. Phys. C 62, 623 (1994).

[30] V. Chernyak, preprints Budker INP 94-69 (1994) [hep-ph/9407353; Budker INP 95-18 (1995) hep-ph/9503208. 
[31] A.F. Falk, H. Georgi, B. Grinstein and M.B. Wise, Nucl. Phys. B 343, 1 (1990).

[32] P. Colangelo and F. De Fazio, preprint BARI-TH/96-230 (1996) hep$\mathrm{ph} / 9604425$.

[33] J.L. Rosner, Phys. Lett. B 379, 267 (1996).

[34] P. Abreu et al. (DELPHI Collaboration), preprint DELPHI 95-107 PHYS 542 (1995), to appear in: Proceedings of the International Europhysics Conference on High Energy Physics, Brussels, Belgium, September 1995.

[35] B. Grzadkowski and W.-S. Hou, Phys. Lett. B 272, 383 (1991).

[36] A.L. Kagan, Phys. Rev. D 51, 6196 (1995).

[37] L. Roszkowski and M. Shifman, Phys. Rev. D 53, 404 (1996).

[38] M. Ciuchini, E. Gabrielli, and G.F. Giudice, preprint CERN-TH/96-73 (1996) hep-ph/9604438.

[39] For a summary of measurements of $B_{\mathrm{SL}}$, see: T. Skwarnicki, preprint hepph/9512395, to appear in: Proceedings of the 17th International Symposium on Lepton Photon Interactions (LP95), Beijing, P.R. China, August 1995.

[40] P. Perret, PCCF-RI 9507 (1995), to appear in: Proceedings of the International Europhysics Conference on High Energy Physics, Brussels, Belgium, September 1995.

[41] H. Simma, G. Eilam and D. Wyler, Nucl. Phys. B 352, 367 (1991).

[42] G. Buchalla, I. Dunietz and H. Yamamoto, Phys. Lett. B 364, 188 (1995).

[43] T. Browder, preprint UH 511-836-95 (1995), to appear in: Proceedings of the International Europhysics Conference on High Energy Physics, Brussels, Belgium, September 1995 hep-ex/9602009.

[44] G. Calderini, presented at the 31th Rencontres de Moriond: QCD and High Energy Hadronic Interactions, Les Arcs, France, March 1996;

D. Buskulic et al. (ALEPH Collaboration), preprint CERN-PPE/96-117 (1996).

[45] S.J. Brodsky, G.P. Lepage, and P.B. Mackenzie, Phys. Rev. D 28, 228 (1983); G.P. Lepage and P.B. Mackenzie, Phys. Rev. D 48, 2250 (1993).

[46] M. Luke, M.J. Savage and M.B. Wise, Phys. Lett. B 343, 329 (1995); 345, 301 (1995). 
[47] K. Honscheid, to appear in: Proceedings of the 20th Johns Hopkins Workshop, Heidelberg, June 1996.

[48] E. Witten, Nucl. Phys. B 160, 57 (1979).

[49] A.J. Buras, J.M. Gérard and R. Rückl, Nucl. Phys. B 268, 16 (1986). 